Grade and Tonnage Data Used to Construct

Models for the Regional Alaskan Mineral Resources

Assessment Program

by

D.A. Singer, W.D. Menzie, J.H. DeYoung, Jr.,

M. Sander $1 /$, and A. Lott

1/ Stanford University, Stanford, California 94305

U.S. Geological Survey

Open-file Report 80-799

This report is preliminary and has not been edited or reviewed for conformity with U.S. Geological Survey standards and nomenciature. 
In early 1978, the U.S. Geological Survey published four regional studies (U.S.G.S. Open-file Reports 78-1A to E) describing the metalliferous mineral resource potential of 80 percent of the State of Alaska. These studies were part of the Regional Alaskan Mineral Resource Assessment Program (RAMRAP). Basic steps in these reports included delineation of areas favorable or permissive for various types of mineral deposits, estimation of the number of deposits of each type within each. delineated area where possible, and for 11 deposit types, construction of grade-tonnage or contained metal models. Because of space and time limitations, documentation of the criteria for the selection of deposits used in the grade-tonnage models, grade and tonnage values, sources of information, and limitations of the grade and tonnage values were not included in the reports; this paper meets these needs.

Tonnages and average grades of incompletely explored deposits are seldom well known and, of course, are not known for unfound deposits. Variation of grades and tonnages of well-explored deposits is quite large but much of this variation is due to differences in deposit types. Within specified deposit types the variation can be much less and can be represented by frequency destributions of grade and tonnage for wel1explored deposits of each type. Frequency distributions of this type were used in the mineral resource reports of Alaska as models of tonnages and average grades of the yet-to-be discovered and incompletely explored deposits. Thus the assertion that an area is geologically favorable for the occurrence of a specified type of mineral deposit can be further defined by quantitative information about the quality and quantity 
of mineral commodities contained in such deposits.

Total tonnages and average grades for known deposits were calculated on the basis of past production plus estimated reserves or, where available, estimated resources. These estimates, plus deposit names, general locations, and data sources are listed in this report in tables for each of the deposit types for which models were constructed (Tables 1-10). Preceding each table is a short description of the criteria used to classify the deposits. Data used in the grade-tonnage or containedmetal models for the following deposit types are presented; podiform chromite, porphyry copper, porphyry molybdenum, skarn tungsten, mercury, nickel in small intrusions, vein gold, mafic volcanogenic sulfide, felsic and intermediate volcanogenic sulfide, and skarn copper. Data sources for each table are listed separately at the end. Location codes are listed in Table 11. Revised grade-tonnage models are presented in Table 12. A discussion of errors that can exist in this type of data is provided in the following section.

\section{Data Errors}

Because the grade-tonnage models are supposed to add information about the mineral resource potential of an area, it is important that models of grades and tonnages be representative of those that occur in the delineated areas. The models may not be representative if the wrong deposit type model is applied to an area, or if the grades or tonnages used to construct the model are not representative of the deposit type. With careful consideration of the geology and known deposits of an area, such as was the case in the mineral resource assessments of Alaska, errors due to misspecification of deposit types tend to be quite small. Errors 
due to non-representative grades or tonnages can be a result of the incorrect values of properly classified deposits or of incorrectly classified deposits used in the models.

A deposit of one type can be placed with a group of deposits of another type through a classification mistake. Combinations of host and associated rock types, contained metals, mineralogy, geologic settings, and comparison with recognized mineral deposits of each type were used to identify deposits that belonged to the various deposit types. Mistakes in classification can be a result of errors in the geologic description of a deposit in the literature or in our interpretation of the geologic description. A few errors of both types probably exist in the data sets presented here. It is unlikely, however, that discovery and correction of these errors would significantly change the grade-tonnage models used in the resource assessments.

Errors related to incorrect deposits used in the models can also be due to unrecognized regional differences in grades and/or tonnages within a mineral deposit type. Regional differences in grades and/or tonnages. are known to exist for porphyry copper deposits and for podiform chromite deposits and may exist but not be recognized for some of the other deposit types.

Incorrect values can come about in several ways and may cause the grade-tonnage model developed from the values to not be representative of the population of interest. Although reporting errors exist in the literature, gross errors are usually detected by plotting the data and by examining data from several sources.

A final source of error is due to the effects of economics on the 
quality of data available. Grade and tonnages estimates are usually based on drilling of the deposits. Because of the expense involved in drilling, mining concerns commonly drill enough holes to prove or disprove the economic viability of the mining the deposit. This process can lead to a tonnage, and in some cases a grade estimate that is biased with respect to the grade and tonnage of the mineralized rock in a deposit. Within individual deposits, tonnage estimates tend to be biased downword and grade estimates biased upward. A related problem is the tendency of lower grade deposits not to be reported because they may not be economic to mine at the time they are explored. Balancing these biases to some extent is the inclusion of past production which, for older deposits at or near depletion, tends to reduce the biases. For the more recently discovered and the larger tonnage deposits, drilling and the resultant estimates are often more complete because mining concerns attempt to develop long-range mine plans now. It is also more common in recent years for governments and private industry to report grades and tonnage estimates of uneconomic deposits.

\section{Acknowledgments}

The data were obtained from a variety of sources and many individuais among them Paul Schruben, Gus Goudarzi, Maureen Johnson, and Jocelyn Peterson. 
Chromite deposits were classiried as podiform if their form was that of lenticular or approximately tabular pods occuring in irregular peridotite masses or peridotite-gabbro complexes of the alpine type. Data used for the RAMRAP podiform chromite model were all from California because of reported similarities of the California deposits to many in Alaska and because of the excellent documentation of the California deposits. Reported grades should be used with caution; they may represent the shipped grade which had been concentrated or the grade in place. A few of the grades and tonnages from the larger deposits represent the combination of several deposits from a district. Reports of multiple grades from different parts of a deposit were handled by recalculating the grades and tonnages to an average grade for the total tonnage. 
Table 1.--Chromite Deposits from California

(data sources 7 isted in references)

Deposit name

$$
\begin{array}{cc}
\text { Location } & \text { Grade } \\
\text { (County) } & \left(\begin{array}{c}
\text { Percent } \\
\left.\mathrm{Cr}_{2} \mathrm{O}_{3}\right)
\end{array}\right.
\end{array}
$$

Tonnage

(Metric tons)
Newmon Mine

Allan (Johnson Lease)

Courtwright (Daggett Lease)

Courtwright

Detert

Ellis

Kremmel and Froelich

Wait

Anti-Axis

Big Bend

Big Pine Claim

Christain Place

Dickey and Drisbach Prospect

Green ridge

Hendricks No. 2

Lambert

Liberty Bond Claim

Little Hope

Mary Jane

North Star (Red Mtn)

Parkeson

Park's Ranch

P.U.P. (Zenith)

Simmons

Stewart

Suzy Bell (Lucky Strike)

Swayne

War Bond

War Eagle and Miller Deposits
Al ameda

Amador

...do...

...do...

...do...

...do...

...do...

...do...

Butte

...do...

$\therefore$ do...

...do...

...do...

...do...

...do...

...do...

...do...

....do...

... do...

...do...

... do...

... do...

...do...

... do...

...do...

...do...

...do...

...do...

...do...
48

40

40

39

38

40

33

38

41

48

32

53

35

35

14

42

28

41

37

37

41

40

36

36

52

38

32

35

13
$1.5 \times 10^{3}$

$6.3 \times 10^{1}$

$6.0 \times 10^{1}$

$1.16 \times 10^{2}$

$1.9 \times 10^{2}$

$6.0 \times 10^{1}$

$3.57 \times 10^{2}$

$2.5 \times 10^{2}$

$3.7 \times 10^{1}$

$9.0 \times 10^{1}$

$2.25 \times 10^{2}$

$3.1 \times 10^{1}$

$3.0 \times 10^{1}$

$9.4 \times 10^{1}$

$8.93 \times 10^{2}$

$3.3 \times 10^{3}$

$3.3 \times 10^{1}$

$8.5 \times 10^{1}$

$4.1 \times 10^{1}$

$1.35 \times 10^{2}$

$3.1 \times 10^{1}$

$5.2 \times 10^{2}$

$1.15 \times 10^{3}$

$7.7 \times 10^{1}$

$1.26 \times 10^{2}$

$3.0 \times 10^{2}$

$9.0 \times 10^{2}$

$5.0 \times 10^{1}$

$2.0 \times 10^{4}$ 
Deposit

Location

(County)

Grade

(percent

$\left.\mathrm{Cr}_{2} \mathrm{O}_{3}\right)$

Tonnage

(Metric tons)

Gillan

Fields and Stoker

Ellingrood

Bowie Estate

American Asbestos Co.

Clary and Langford

Holbrook and McGuire

Liberty

Madrid

Mayflower

Maxwel1

Valenti

Vogelgesang

Walker

Ward and Lyons

Alyce and Blue Jay Claims

Apex

Big Dipper (Robr)

Bonanza

Chrome Hill No 1

Coon Mt. Nos. 1-3

Copper Creek (Low divide)

Fourth of July

French $\mathrm{Hill}$

High Dome

High Plateau

Judy (Hicks)

Muzzleloader (Stevens No.1)

Lone Gravel

Mt. View

Rattlesnake Mt.

Richey, U.S. and S.J.
Calaveras

...do...

...do...

...do...

...do...

...do...

...do...

...do...

...do...

...do...

...do...

... do...

...do...

...do...

Del Nlorte

...do...

...do...

...do...

....do...

...do...

...do...

...do...

...do...

...do...

...do...

... do...

...do...

...do...

...do...

...do...

...do...

...do...
33

43

38

37

35

46

47

48

32

48

47

28

44

40

31

49

38

42

51

46

40

41

38

45

45

53

44

50

42

42

42

40
$1.4 \times 10^{2}$

$2.12 \times 10^{2}$

$5.0 \times 10^{2}$

$4.0 \times 10^{2}$

$1.0 \times 10^{2}$

$3.48 \times 10^{2}$

$8.5 \times 10^{2}$

$3.25 \times 10^{2}$

$1.4 \times 10^{3}$

$6.67 \times 10^{2}$

$3.3 \times 10^{1}$

$2.0 \times 10^{2}$

$2.38 \times 10^{2}$

$7.1 \times 10^{1}$

$2.0 \times 10^{3}$

$4.0 \times 10^{1}$

$1.0 \times 10^{1}$

$3.39 \times 10^{2}$

$1.49 \times 10^{2}$

$2.0 \times 10^{1}$

$5.7 \times 10^{1}$

$3.35 \times 10^{3}$

$4.0 \times 10^{2}$

$2.04 \times 10^{4}$

$2.95 \times 10^{2}$

$1.5 \times 10^{4}$

$3.85 \times 10^{2}$

$1.69 \times 10^{2}$

$2.8 \times 10^{2}$

$9.35 \times 10^{3}$

$3.36 \times 10^{3}$

$2.3 \times 10^{1}$ 
Deposit

Location

(County)

Grade

(Percent

$\left.\mathrm{Cr}_{2} \mathrm{O}_{3}\right)$
St. Patrick (Camp 8)

Skyline Mine

Sunrise

Toujours Gai

White Feather

Apex

Darrington

Gold Bug Claims

Green Mine

Helemar

Hill-Top Chrome

Hoff

Murphy

Pillikin

Shelly

Sheppard Mine

Simon

Stafford

Alice Mine

Black Bow Claim (Avery)

Blue Brush

Butler, Estate chrome, etc.

Camden Mine

Clara $\mathrm{H}$

Chrome Gulch Group

Jack Sprat Group

Lacey

Letty

Long Ledge Group

Lotty

Little Rock Mine

Lucky Boy
Del Norte

...do...

...do...

... do...

...do...

El Dorado

...do...

...do...

...do...

...do...

... do...

...do...

... do...

...do...

...do...

...do...

...do...

...do...

Fresno

...do...

...do...

... do...

....do...

....do...

... do...

... do...

....do...

...do...

...do...

...do...

...do...

...do...
40

47

51

47

38

45

45

40

47

38

45

31

10

43

30

34

35

45

40

29

42

46

41

43

40

30

44

44

10

44

25

34
Tonnage

(Metric tons)

$1.27 \times 10^{2}$

$1.2 \times 10^{3}$

$4.89 \times 10^{2}$

$2.64 \times 10^{2}$

$6.5 \times 10^{1}$

$8.0 \times 10^{0}$

$2.2 \times 10^{4}$

$2.0 \times 10^{2}$

$1.5 \times 10^{2}$

$8.7 \times 10^{1}$

$9.0 \times 10^{0}$

$2.0 \times 10^{2}$

$5.0 \times 10^{3}$

$1.1 \times 10^{5}$

$1.28 \times 10^{3}$

$1.2 \times 10^{2}$

$1.05 \times 10^{2}$

$1.98 \times 10^{2}$

$4.5 \times 10^{1}$

$1.8 \times 10^{1}$

2.7 c $10^{1}$

$2.3 \times 10^{4}$

$1.2 \times 10^{2}$

$6.65 \times 10^{3}$

$3.0 \times 10^{2}$

$3.19 \times 10^{3}$

$3.73 \times 10^{3}$

$2.0 \times 10^{1}$

$9.2 \times 10^{3}$

$1.5 \times 10^{1}$

$2.0 \times 10^{1}$

$1.2 \times 10^{2}$ 
Deposit name

Location

(County)

Grade

(Percent

$\left.\mathrm{Cr}_{2} \mathrm{O}_{3}\right)$
Manchester Mine

McCurty

Red Slide Group

Rock Wren Mine

Rose Claims

Saint

Sunset

Black Diamond or Grey Eagle Group

Binder No. 1

Blue Creek Tunnel

Lassic Peak

Pecwan

Pyramid

White Cedar

Wilder (Fish Creek)

Black Bart (Great Western)

Butler Claims

Gunn Claims

Harp and Sons Ranch Deposits

Kangaroo Court Mines

Sutro Mine

Lucky Strike Mine

Daisy (Aldelabron)

Elder Claim

Moore Mine

Mullaly Mine

Nichel ini Mine

Alta $\mathrm{Hill}$

Bowden Prospect

Codd Prospect

Dickerson

Eden

\section{Fresno}

...do...

....do...

... do...

...do...

... do...

...do...

Glenn

Humboldt

...do...

... do...

...do...

...do...

...do...

...do....

Lake

...do...

...do...

...do...

...do...

...do...

...do...

Monterey

Napa

...do...

...do...

...do...

Nevada

...do...

...do...

...do...

...do...

44

35

42

15

35

44

41

46

46

46

47

42

45

45

40

48

45

38

51

40

43

48

47

45

40

47

45

34

18

15

35

25
$3.5 \times 10^{1}$

$3.14 \times 10^{2}$

$2.5 \times 10^{2}$

$1.25 \times 10^{3}$

$1.2 \times 10^{2}$

$1.27 \times 10^{2}$

$1.0 \times 10^{2}$

$3.96 \times 10^{4}$

$5.0 \times 10^{0}$

$2.4 \times 10^{1}$

$3.0 \times 10^{0}$

$7.3 \times 10^{1}$

$7.6 \times 10^{1}$

$2.85 \times 10^{2}$

$1.45 \times 10^{2}$

$4.0 \times 10^{2}$

$1.6 \times 10^{1}$

$2.4 \times 10^{1}$

$5.0 \times 10^{1}$

$1.5 \times 10^{1}$

$1.2 \times 10^{1}$

$1.5 \times 10^{3}$

$3.0 \times 10^{2}$

$2.0 \times 10^{1}$

$44.6 \times 10^{2}$

$8.5 \times 10^{1}$

$2.0 \times 10^{3}$

$1.77 \times 10^{2}$

$1.0 \times 10^{2}$

$7.1 \times 10^{1}$

$6.0 \times 10^{0}$

$5.0 \times 10^{0}$ 
Deposit name

Location

(County)

Grade

(Percent

$\left.\mathrm{Cr}_{2} \mathrm{O}_{3}\right)$

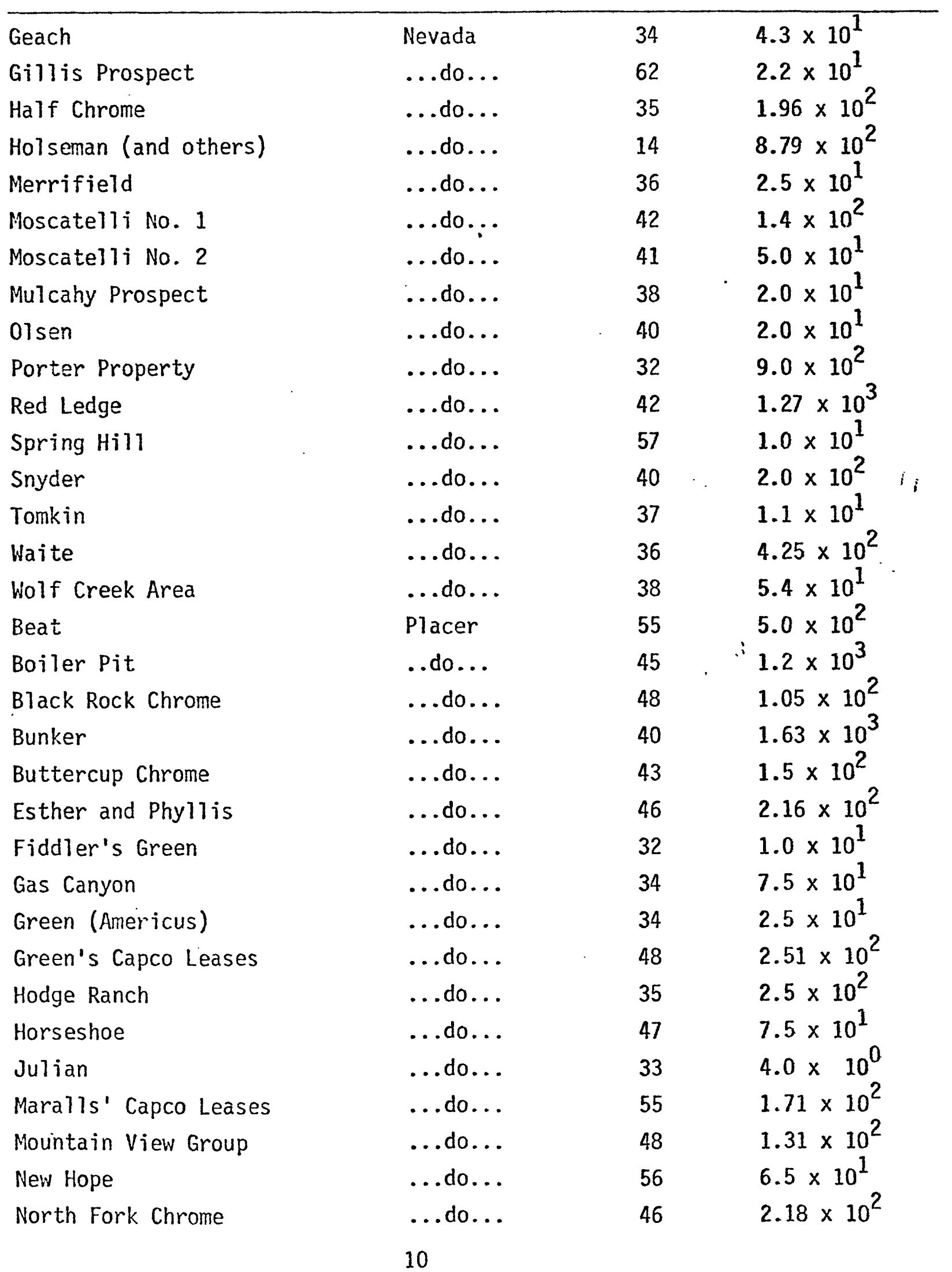


Grade

(Percent

$\mathrm{Cr}_{2} \mathrm{O}_{3}$ )

\begin{tabular}{|c|c|c|c|}
\hline Parker & Placer & 11 & $5.5 \times 10^{3}$ \\
\hline Poco Tiempo Quartz & ...do... & 40 & $3.5 \times 10^{1}$ \\
\hline Randa11 & ...do... & 51 & $5.5 \times 10^{1}$ \\
\hline Snakehead (Jumbo) & ...do... & 47 & $6.5 \times 10^{1}$ \\
\hline Southern Pacific property & ...do... & 39 & $1.8 \times 10^{2}$ \\
\hline Sunset & ...do... & 55 & $5.61 \times 10^{2}$ \\
\hline Washout & ...do... & 45 & $4.71 \times 10^{2}$ \\
\hline West Chrome & ...do... & 45 & $2.82 \times 10^{2}$ \\
\hline Cattle Springs & Plumas & 39 & $7.15 \times 10^{1}$ \\
\hline Chicago & ...do... & 42 & $1.6 \times 10^{1}$ \\
\hline Clover Leaf & ...do... & 43 & $2.5 \times 10^{1}$ \\
\hline Commander & ...do... & 39 & $2.3 \times 10^{1}$ \\
\hline Diamond & $\because$. do... & 46 & $2.2 \times 10^{1}$ \\
\hline Edeline & ...do... & 47 & $6.0 \times 10^{0}$ \\
\hline Hudson (Fuller Claims) & ...do... & 45 & $1.1 \times 10^{1}$ \\
\hline Jack Forth & ...do... & 36 & $4.2 \times 10^{1}$ \\
\hline Mc Carty & ...do... & 40 & $2.0 \times 10^{2}$ \\
\hline Poodle Dog & ...do... & 37 & $2.2 \times 10^{1}$ \\
\hline Skyline No. 1 & ...do... & 43 & $1.2 \times 10^{1}$ \\
\hline Skyl ine No. 2 & ...do... & 46 & $3.8 \times 10^{1}$ \\
\hline Spot & ...do... & 42 & $6.0 \times 10^{1}$ \\
\hline White Pine Mine & ...do... & 49 & $5.5 \times 10^{2}$ \\
\hline Wolf Creek & ...do... & 32 & $2.4 \times 10^{2}$ \\
\hline Western Magnesite & Santa Clara & 35 & $2.0 \times 10^{1}$ \\
\hline El Primero & San Luis Obispo & 30 & $3.25 \times 10^{2}$ \\
\hline Castro Mine & ...do... & 43 & $4.93 \times 10^{4}$ \\
\hline Zerfirg Ranch & ...do... & 46 & $1.0 \times 10^{1}$ \\
\hline Pine Mt. Claims & ...do & 38 & $8.5 \times 10^{1}$ \\
\hline Trinidad & ...do... & 45 & $1.6 \times 10^{3}$ \\
\hline Sousa Ranch & ...do... & 45 & $8.3 \times 10^{1}$ \\
\hline Norcross & ...do... & 50 & $2.49 \times 10^{3}$ \\
\hline Lucky Strike Mine & ...do... & 43 & $8.3 \times 10^{1}$ \\
\hline Victory No. 3 & ...do... & 41 & $2.8 \times 10^{1}$ \\
\hline
\end{tabular}




\begin{tabular}{|c|c|c|c|}
\hline Deposit name & $\begin{array}{l}\text { Location } \\
\text { (County) }\end{array}$ & $\begin{array}{c}\text { Grade } \\
\text { (Percent } \\
\left.\mathrm{Cr}_{2} \mathrm{O}_{3}\right)\end{array}$ & $\begin{array}{c}\text { Tonnage } \\
\text { (Metric tons) }\end{array}$ \\
\hline Sweetwater & San Luis Obispo & 46 & $1.04 \times 10^{3}$ \\
\hline Sunshine & ...do... & 38 & $3.9 \times 10^{1}$ \\
\hline (unnamed) & ...do... & 51 & $2.1 \times 10^{3}$ \\
\hline Shotgun Creek & Shasta & 44 & $2.99 \times 10^{3}$ \\
\hline Round Bottom & ...do... & 49 & $4.71 \times 10^{2}$ \\
\hline Little Castle Creek Mine & ...do... & 46 & $1.67 \times 10^{4}$ \\
\hline Forest Queen & ...do... & 43 & $1.88 \times 10^{3}$ \\
\hline Dorriss & Sierra & 45 & $7.5 \times 10^{1}$ \\
\hline Finan & ...do... & 47 & $8.0 \times 10^{0}$ \\
\hline Gibsonville & ...do... & 44 & $2.62 \times 10^{2}$ \\
\hline Golconda Fraction & $\ldots$ do... & 39 & $1.2 \times 10^{2}$ \\
\hline Miltion & ...do... & 41 & $2.5 \times 10^{2}$ \\
\hline Oxford & ...do... & 38 & $4.0 \times 10^{3}$ \\
\hline Roupe & ...do... & 44 & $4.0 \times 10^{2}$ \\
\hline White Bear & ...do... & 41 & $3.75 \times 10^{2}$ \\
\hline Coggins & Siskiyou & 39 & $4.22 \times 10^{3}$ \\
\hline Cyclone Gap & ...do... & 47 & $2.4 \times 10^{3}$ \\
\hline Doe Flat & ...do... & 36 & $2.4 \times 10^{3}$ \\
\hline Dozier & ...do... & 42 & $4.65 \times 10^{2}$ \\
\hline Elk Creek Claim & ...do... & 41 & $1.87 \times 10^{2}$ \\
\hline Fairview & ...do... & 39 & $2.26 \times 10^{3}$ \\
\hline Hayden and Hilt & ...do... & 50 & $6.9 \times 10^{1}$ \\
\hline Josephine & ...do... & 45 & $1.0 \times 10^{3}$ \\
\hline McGuffy Creek Group & ...do... & 55 & $8.25 \times 10^{3}$ \\
\hline Moffett Creek Group & ...do... & 49 & $4.25 \times 10^{2}$ \\
\hline Peg Leg (Lambert) & ...do... & 54 & $6.31 \times 10^{2}$ \\
\hline Seiad Creek (Mt. View) & ...do... & 36 & $6.0 \times 10^{4}$ \\
\hline Snowy Ridge & ...do... & 39 & $2.98 \times 10^{2}$ \\
\hline Laton Mine & Sonoma & 48 & $3.0 \times 10^{3}$ \\
\hline Madeira & ...do... & 34 & $1.0 \times 10^{2}$ \\
\hline Meeker (Sonoma Chrome, Inc) & ...do... & 40 & $2.0 \times 10^{3}$ \\
\hline Welch Prospect & ...do... & 43 & $2.0 \times 10^{1}$ \\
\hline
\end{tabular}


Deposit name

Location

Grade

(County)

(Percent

$\mathrm{Cr}_{2} \mathrm{O}_{3}$ )

\begin{tabular}{|c|c|c|c|}
\hline No 5 & Stanislaus & 51 & $9.95 \times 10^{2}$ \\
\hline Lucky Girl Mine & ...do... & 42 & $1.5 \times 10^{2}$ \\
\hline Chrome Camp Mine & ...do... & 40 & $9.3 \times 10^{2}$ \\
\hline Black Bear Mine & ...do... & 50 & $3.42 \times 10^{2}$ \\
\hline Black Bart Group & ...do... & 35 & $4.14 \times 10^{2}$ \\
\hline Adobe Canyon Group & ...do... & 27 & $5.0 \times 10^{2}$ \\
\hline Black Chrome & Tehama & 49 & $4.4 \times 10^{1}$ \\
\hline Blue Sky (Lucky Star) & ...do... & 47 & $4.52 \times 10^{2}$ \\
\hline Elder Creek & ...do... & 46 & $8.4 \times 10^{1}$ \\
\hline Elder Creek Group & ...do... & 45 & $3.53 \times 10^{3}$ \\
\hline KTeinsorge Group & ...do... & 46 & $1.64 \times 10^{3}$ \\
\hline Noble Electric Co. & ... do... & 47 & $1.8 \times 10^{3}$ \\
\hline State School & ...do... & 47 & $6.74 \times 10^{2}$ \\
\hline Bragdor & Trinity & 46 & $1.0 \times 10^{2}$ \\
\hline Crow Creek Group & ...do... & 41 & $2.0 \times 10^{3}$ \\
\hline Happy Go Lucky & ...do... & 45 & $4.5 \times 10^{1}$ \\
\hline I Wonder & ...do... & 52 & $1.2 \times 10^{1}$ \\
\hline Mule Creek & ...do... & 44 & $3.3 \times 10^{1}$ \\
\hline Oak Ridge & ...do... & 49 & $1.7 \times 10^{1}$ \\
\hline Redskin & ...do... & 42 & $1.0 \times 10^{2}$ \\
\hline September Morn & ...do... & 48 & $6.4 \times 10^{1}$ \\
\hline Shamrock & ...do... & 43 & $5.0 \times 10^{1}$ \\
\hline Stark Bee & ...do... & 44 & $4.6 \times 10^{1}$ \\
\hline Sunnys Tope & ...do... & 39 & $2.6 \times 10^{2}$ \\
\hline Yellow Pine & ...do... & 50 & $2.1 \times 10^{2}$ \\
\hline Tangle Blue Divide & ...do... & 45 & $9.7 \times 10^{1}$ \\
\hline Earl Smith & Tulare & 39 & $2.55 \times 10^{2}$ \\
\hline Gill (Gill Ranch) & ...do... & 40 & $4.0 \times 10^{2}$ \\
\hline Holston (Vaughn) & ...do... & 47 & $3.33 \times 10^{3}$ \\
\hline Ace of Spades & Tuolumne & 21 & $4.35 \times 10^{2}$ \\
\hline Booker Lease & ...do... & 36 & $4.3 \times 10^{1}$ \\
\hline Don Pedro & ...do... & 40 & $6.0 \times 10^{2}$ \\
\hline Eggling and Williams & ...do... & 38 & $3.63 \times 10^{2}$ \\
\hline
\end{tabular}




\begin{tabular}{llcc}
\multicolumn{1}{c}{ Deposit name } & $\begin{array}{c}\text { Location } \\
\text { (County) }\end{array}$ & $\begin{array}{c}\text { Grade } \\
\left(\mathrm{Percent}^{\mathrm{Cr}} \mathrm{O}_{3}\right)\end{array}$ & $\begin{array}{c}\text { Tonnage } \\
\text { (Metric tons) }\end{array}$ \\
\hline Mc Cormick & Tuolumne & 46 & $3.85 \times 10^{3}$ \\
Mackay & $\ldots$ do... & 37 & $4.15 \times 10^{2}$ \\
Mum and Alice June claims & $\ldots$ do... & 17 & $2.5 \times 10^{4}$ \\
North End, West End and & $\ldots$ do... & 38 & $3.5 \times 10^{3}$ \\
$\quad$ Spotted Fawn & $\ldots$ do... & 33 & $5.0 \times 10^{1}$ \\
Perconi Ranch Deposits & $\ldots$ do... & 35 & $1.70 \times 10^{3}$ \\
Quigg & $\ldots$ do... & 23 & $8.45 \times 10^{3}$ \\
Richards & $\ldots$ do... & 31 & $8.5 \times 10^{0}$ \\
Shafer lease & $\ldots$ do... & 37 & $2.9 \times 10^{1}$ \\
Sims & $\ldots$ do... & 31 & $8.5 \times 10^{2}$ \\
Sullivan and Kahl & $\ldots$ do... & 33 & $1.75 \times 10^{1}$ \\
Challenge Area & $\ldots$ do... & 38 & $3.86 \times 10^{1}$ \\
Camptonville Area & & &
\end{tabular}




\section{Porphyry Copper}

Deposits were placed in the class if they included disseminated pyrite, chalcopyrite, and frequently molybdenite and bornite in or near felsic to intermediate intrusive rocks. Most deposits had at least one porphyry facies of intrusive. In most cases, deposits were placed in this class if they were called porphyries in the literature. Deposits from western Canada and Alaska were selected as the most appropriate model for Alaskan deposits because the average grades and tonnages of porphyry copper deposits tend to be lower for deposits located farther north along the trend of deposits from Chile, through the southwestern U.S., to British Columbia and ATaska. In spite of this precaution, the grades of the Canadian deposits appeared to be too high compared to those reported in preliminary drilling of the Alaskan deposits; the grade model used in the RAMRAP reports was therefore a result of a subjective adjustment of the observed distribution. Both grade models are presented in Table 12. 
Table 2.--Porphyry Copper Deposits

Deposit name $\begin{array}{cl}\text { Locational } & \begin{array}{c}\text { Copper Molybdenum Tonnage Reference } \\ \text { grade }\end{array} \text { grade } \\ \text { (Percent) (Percent) tons } & \text { (Metric }\end{array}$

tons
$\times 10^{6}$ )

\begin{tabular}{|c|c|c|c|c|c|}
\hline Bell Copper & CNBC & 0.48 & 0.006 & 100. & 3 \\
\hline Morrison & CNBC & .42 & .013 & 95. & 3 \\
\hline Dorothy & CNBC & .25 & .01 & 45. & 5 \\
\hline Krain (Keystone) & CNBC & .45 & .013 & 18. & 1 \\
\hline Axe & CNBC & .45 & .012 & 45.4 & 2 \\
\hline Ann (Gravel Mt.) & CNBC & .27 & .003 & 43.4 & 2 \\
\hline Poison Mountain & CNBC & .33 & .015 & 175. & 3 \\
\hline Berg & CNBC & .40 & .03 & 363. & 2 \\
\hline Schaft Creek (Laird) & CNBC & .40 & .023 & 330. & 3 \\
\hline Paramount Mining & CNBC & .337 & .028 & 90.7 & 1 \\
\hline Patton Hill (Casino) & CNYU & .37 & .0234 & 162. & $2^{i}$ \\
\hline Highmount (Ide) & CNBC & .285 & .031 & 136. & 1 \\
\hline Bethlehem (all properties) & CNBC & .46 & .013 & 900. & 8 \\
\hline Brenda & CNBC & .18 & .05 & 159. & 3 \\
\hline Gilbraltor & CNBC & .37 & .01 & 327. & 3 \\
\hline Maggie & CNBC & .28 & .031 & 180. & 3 \\
\hline Huckleberry & CNBC & .401 & .013 & 78.9 & 3 \\
\hline Lornex & CNBC & .41 & .013 & 425. & 3 \\
\hline Island Copper & CNBC & .52 & .019 & 257. & 3 \\
\hline Caribou & CNBC & $.50^{\circ}$ & .025 & 37. & 1 \\
\hline Bond Creek & USAK & .30 & .019 & 500. & 6 \\
\hline Orange $\mathrm{Hill}$ & USAK & .35 & .02 & 320. & 6 \\
\hline $0 \mathrm{~K}$ & CNBC & .30 & .01 & 68. & 3 \\
\hline Afton & CNBC & .90 & & 71. & 7 \\
\hline Granisle & CNBC & .43 & & 85. & 3 \\
\hline Ingerbeil( + Similkameen) & CNBC & .53 & & 68. & 4 \\
\hline Galore (Stikine) & CNBC & 1.00 & & 125. & 1 \\
\hline Valley Copper & CNBC & .48 & & 900. & 2 \\
\hline Sam Goosly & CNBC & .33 & & 40. & 2 \\
\hline Redgroup & CNBC & .56 & & 41. & 1 \\
\hline
\end{tabular}


Deposit name

Locationa/ Copper Molybdenum Tonnage Reference grade (Percent) (Percent) tons grade (Metric

\begin{tabular}{|c|c|c|c|c|c|}
\hline \multirow{3}{*}{$\begin{array}{l}\text { Evening Star \& Galaxy copper } \\
\text { June (Dease Lake) }\end{array}$} & & \multicolumn{4}{|c|}{$\left.\times 10^{\circ}\right)$} \\
\hline & CNBC & .58 & & 5.4 & 1 \\
\hline & CNBC & .389 & & 30. & 1 \\
\hline Primer & CNBC & .20 & & 23. & 5 \\
\hline Kwanika & CNBC & .20 & & 36. & 5 \\
\hline Big Onion & CNBC & .36 & & 18. & 5 \\
\hline Catface & CNBC & .47 & & 200. & 1 \\
\hline Lorraine & CNBC & .70 & & 10. & 3 \\
\hline Wheal Tamar (Ajax) & CNBC & .50 & & 9. & 1 \\
\hline Copper Mtn. & CNBC & .41 & & 140. & 9 \\
\hline Eagle & CNBC & .50 & .009 & 272. & 10 \\
\hline Gambier Is. & CNBC & .28 & & 297. & 11 \\
\hline
\end{tabular}


Deposits were classified in this group if molybdenite occurs as disseminated grains or stockworks of quartz veins and veinlets in fractured or brecciated, altered granitic intrusive rocks and the associated country rocks or if deposits were reported to be of the porphyry molybdenum type but no information on the geology was presented. Subsequent to the collection of these data and their use in the RAMRAP estimates, rapid developments have occurred in exploration for and knowledge about molybdenum deposits. Several deposits, such as Quartz Hill and Thompson Creek, are now known to be much larger than our original data showed. According to T. Theodore (personal communication, 1979), theoretical considerations resulting from recent research suggest that deposits in Colorado may be of a special type not likely to occur in Alaska. In Table 12, a revised grade-tonnage model that reflects the revised grade and tonnage estimates. and the deletion of Colorado and New Mexico deposits, is presented. 
Table 3.--Porphyry Molybdenum Deposits

\begin{tabular}{|c|c|c|c|c|}
\hline Deposit name a/ & Location $\underline{b}$ & $\begin{array}{l}\text { Molybdenum } \\
\text { grade } \\
\text { (Percent) }\end{array}$ & $\begin{array}{l}\text { Tonnage } \\
\text { (Metric tons } \\
\left.\times 10^{6}\right)\end{array}$ & References \\
\hline Climax* & USCO & 0.20 & 800 & 7 \\
\hline Henderson* & USCO & .297 & 342. & 7 \\
\hline Questa* & USAM & .10 & 230 & 4 \\
\hline Quartz Hill & USAK & .081 & 1180. & 1. \\
\hline Mt. Emmons* & USCO & .26 & 150 & $10^{\circ}$ \\
\hline Endako & CNBC & .86 & 240. & 7 \\
\hline Boss Mountain & CNBC & .22 & 7.6 & 9 \\
\hline Alice (Lime Creek, BC Moly) & CNBC & .12 & 105. & 11 \\
\hline Moly (Bel1) & NCBC & .066 & 32.5 & 2 \\
\hline Adera-Ruby Creek (Adanac) & CNBC & .096 & 94.3 & 2 \\
\hline St. Elmo & CNBC & .20 & 0.07 & 2 \\
\hline Giant & CNBC & .169 & 0.98 & 2 \\
\hline Canam & CNBC & .033 & 1.93 & 2 \\
\hline Elk & CNBC & .139 & 1.93 & 2 \\
\hline Glacier Gulch & CNBC & .17 & 90.7 & 2 \\
\hline Sunshine Creek (combined) & CNBC & .098 & 8.47 & 2 \\
\hline Haskin Mountain & CIBBC & .09 & 12.2 & 2 \\
\hline Carmi \& Lake & CNBC & .09 & 36.3 & 2 \\
\hline Gem (Bailey, Meg) & CNBC & .16 & 27.2 & 2 \\
\hline Lucky Ship & CNBC & .084 & 17.8 & 2 \\
\hline Karen & CNBC & .16 & 54.4 & 2 \\
\hline Roundy Creek & CNBBC & .084 & 7.2 & 3 \\
\hline Red Mountain & CNBC & .24 & 1.0 & 6 \\
\hline Red Bird & CNBC & .15 & 27. & 6 \\
\hline Pitman (JB) & CivBC & .08 & 3.4 & 6 \\
\hline Ajax & CNBC & .073 & 145. & 9 \\
\hline Storie Moly & CNBC & .072 & 45 & 6 \\
\hline Thompson Creek & USID & .11 & 233. & 9 \\
\hline Malmbjerg & grld & .15 & 120. & 8 \\
\hline Cannivan G & USMT & .096 & 180. & 8 \\
\hline Mt. Tolman & USWA & .063 & 816 & 5 \\
\hline
\end{tabular}


Tungsten-bearing deposits were placed in this class if they occurred near the contact of limestone or dolomite with intrusive igneous rocks. The tactite minerals include garnet, epidote, and magnetite, and the tungsten occurs only as scheelite. A variety of data sources were used, including some company-confidential sources. Some of the deposits are listed without deposit names, localities, or sources in order to preserve the confidential nature of the data. The geographic distribution of the deposits is world-wide, but the unidentified deposits are located in the United States. 
Table 4.--Skarn/Tactite Tungsten Deposits

\begin{tabular}{|c|c|c|c|c|}
\hline Deposit name & Locationa/ & $\begin{array}{l}\text { Tungsten } \\
\text { grade } \\
\text { (Percent W) }\end{array}$ & $\begin{array}{l}\text { Tonnage } \\
\text { (Metric } \\
\text { tons } \\
\times 10^{3} \text { ) } \\
\end{array}$ & Reference \\
\hline & & 0.79 & 201. & \\
\hline & & 1.43 & 26. & \\
\hline & & .87 & 128. & \\
\hline & & .32 & 706. & \\
\hline & & .20 & 403. & \\
\hline & & .99 & 329. & \\
\hline & & .56 & 288. & \\
\hline Mactung & CNNT & .71 & 27,000 & 1,2 \\
\hline Tempiute & USNV & .40 & 7,200 & 11 \\
\hline A Tungsten deposit & VNZL & .50 & 1,180 & 5 \\
\hline Quixaba & BRSL & .72 & 163. & 5 \\
\hline Maykhura & URTD & .32 & 708. & 5 \\
\hline King Island & AUTS & .43 & 4,770 & 5 \\
\hline Jersey-Emerald & CNBC & .72 & 758. & 5 \\
\hline Osgood Mountains & USNV & .40 & 1,360 & 5 \\
\hline Starbright & USCA & 1.40 & 9.9 & 5 \\
\hline Tyrny-Auz & URRS & .32 & 21,000 & 5 \\
\hline \multirow[t]{2}{*}{ Brejui } & BRSL & .47 & 3,730 & 8 \\
\hline & & .29 & 916. & \\
\hline Red Button & USMT & .87 & 158. & 3 \\
\hline Iron Mountain & USNM & .87 & 3.2 & 10 \\
\hline San Dong & KORA & 1.35 & 3,400 . & 7 \\
\hline \multirow[t]{2}{*}{ Uludag } & TRKY & .40 & 14,200 & 9 \\
\hline & & .50 & 13,000 & \\
\hline \multirow[t]{4}{*}{ Lost Creek } & USMT & .12 & 2,300 & 4 \\
\hline & & .40 & 3,850 . & \\
\hline & & .55 & 780 & \\
\hline & & .84 & 270. & \\
\hline Cupric & USUT & .28 & 3.6 & 6 \\
\hline
\end{tabular}




\begin{tabular}{lcccc} 
Deposit name & Locationaf & $\begin{array}{l}\text { Tungsten } \\
\text { grade } \\
\text { (Percent W) }\end{array}$ & $\begin{array}{l}\text { Tonnage } \\
\text { (Metric } \\
\text { tons } \\
\times 10^{3} \text { ) }\end{array}$ & Reference \\
\hline Ysxjoberg & SWDN & .26 & $2,500$. & 12 \\
Victory $W$ & CNBC & .40 & 83.8 & 8
\end{tabular}

a/ See Table 11 for Tocation codes. 


\section{Mercury}

Deposits were selected for the RAMRAP mercury model on the basis of recommenations of geologists familiar with the mercury deposits of Alaska. For example, Sainsbury and Mackevett (1965) state: "The Alaskan deposits, therefore, may be expected to be roughly comparable to similar deposits in Nevada in general ways." Similarly, deposits from California were not selected for the model on the basis of informed recommendations. For mercury deposits, data on grades and tonnages is not generally reportad, though the contained metal of the deposits is.

Data for this model were obtained through the efforts of M. Johnson and J. Peterson from the U.S. Geological Survey CRIB file (Peterson and others, 1977), and is available to the public through the G.E. timesharing system. 
Table 5.--Mercury Deposits

(data source listed in references)

\begin{tabular}{|c|c|c|}
\hline Deposit name & Locationa/ & $\begin{array}{l}\text { Mercury content } \\
\text { (Metric tons) }\end{array}$ \\
\hline$A \& B$ & USNV & 7.9 \\
\hline Alexander Mine & USOR & .21 \\
\hline Allen Mine & USNV & 1.7 \\
\hline Allison Prospect & USOR & .10 \\
\hline Alpine Prospect & USNV & .034 \\
\hline Ames \& Bancroft & USOR & .24 \\
\hline Amity & USOR & 11. \\
\hline Andies Property & USNV & 120. \\
\hline Angel Peak & USOR & 1.2 \\
\hline Antelope & USNV & .14 \\
\hline Axelandle & USOR & 210 \\
\hline$B \& B$ & USNV & 7.0 \\
\hline Baldwin & USNV & 4.8 \\
\hline Barnes Butte & USOR & 1.0 \\
\hline Barnum - McDonnell & USWA & 940. \\
\hline Beowaw & USNV & 5.1 \\
\hline Berry Creek & USNV & .034 \\
\hline Betty Mine & USNV & 3.9 \\
\hline Birthday Group & USNV & 9.3 \\
\hline Black Butte & USOR & 660. \\
\hline Black Hawk & USNV & 2.4 \\
\hline Black Lizard & USNV & .17 \\
\hline Blue Can & USNV & 60. \\
\hline Blue Ridge & USOR & 9.3 \\
\hline Bonanza & USOR & 1400 \\
\hline Bonita & USOR & .24 \\
\hline Bretz & USOR & 590. \\
\hline Buckskin & USNV & .069 \\
\hline Buckskin Peak & USNV & 110. \\
\hline Buena Vista & USOR & .31 \\
\hline
\end{tabular}




\begin{tabular}{lll}
\multicolumn{1}{c}{ Deposit name } & LoCational & Mercury content \\
(Metric tons)
\end{tabular}




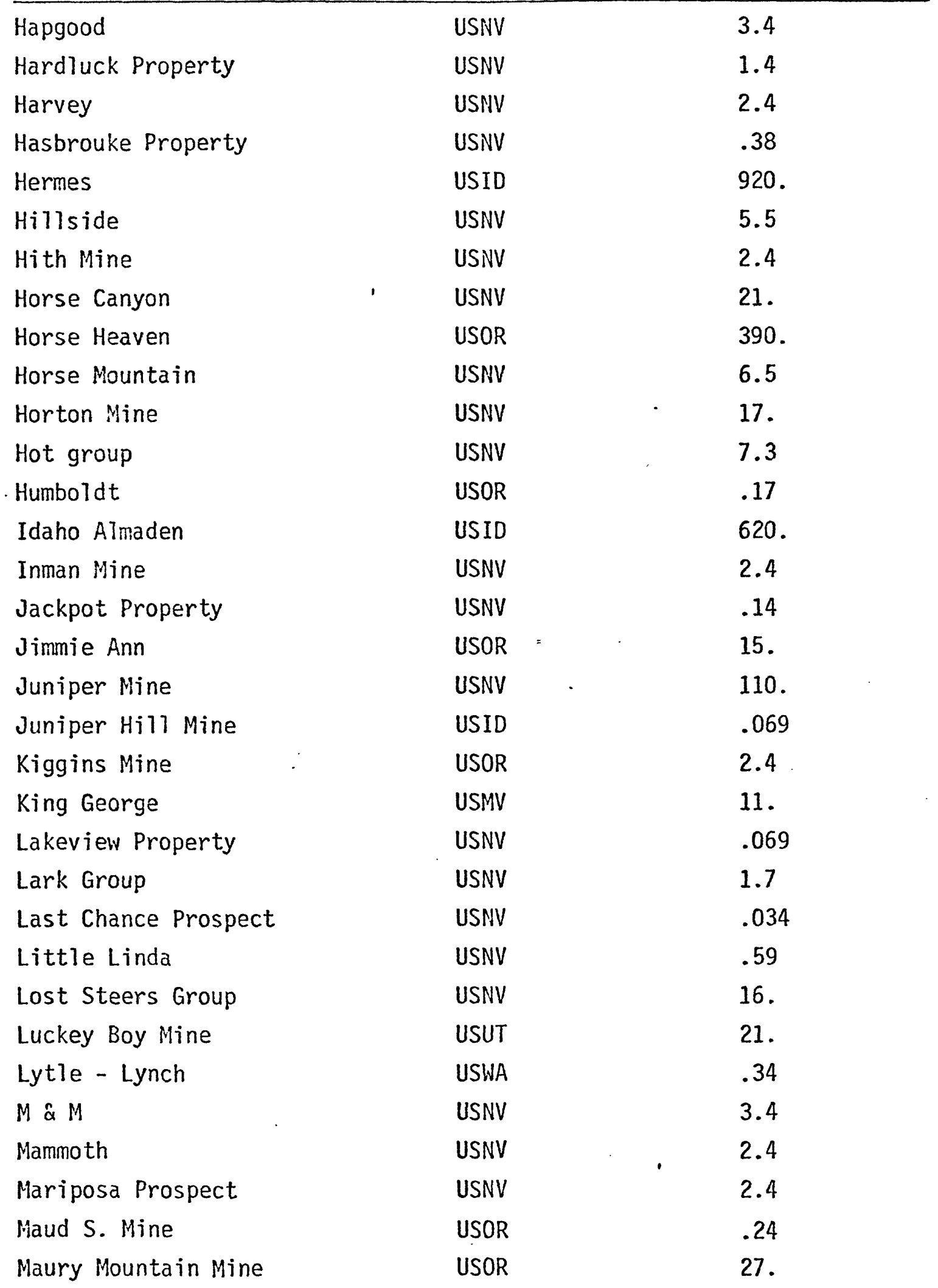




\begin{tabular}{|c|c|c|}
\hline Deposit name & Locationa/ & $\begin{array}{l}\text { Mercury content } \\
\text { (Hetric tons) }\end{array}$ \\
\hline McAdoo & USNV & 57. \\
\hline McCoy & USNV & 11. \\
\hline McDermitt & USNV & 15000. \\
\hline Miller & USID & .034 \\
\hline Mina Development & USNV & 95. \\
\hline Mogul Group & USOR & 1.0 \\
\hline Montgomery & USNV & 17. \\
\hline Moser & USNV & .069 \\
\hline Motherlode Mine & USOR & 21. \\
\hline Mount Tobin & USNV & 51. \\
\hline Mountain King & USOR & 3.3 \\
\hline Nevada Cinnabar & USNV & 220. \\
\hline Nevada Sulfur & USNV & 2.4 \\
\hline Neibuhr Prospect & USNV & 1.4 \\
\hline Nisbet Mine & USOR & 3.5 \\
\hline Nonpareil & USOR & 24. \\
\hline North Fork & USNV & .45 \\
\hline O.K. Prospect & USNV & .34 \\
\hline 0ld Timer Property & USNV & .069 \\
\hline Opalite Mine & USOR & 430 \\
\hline Oronogo & USOR & .45 \\
\hline Pershing Mine & USNV & 150. \\
\hline Platner & USOR & 1.6 \\
\hline Poinsetta Mine & USNV & .34 \\
\hline Probert Mine & USUT & 3.4 \\
\hline Rabbit Hole & USOR & .034 \\
\hline Rat Hole & USNV & .17 \\
\hline Rattlesnake & USNV & .45 \\
\hline Red Bird Group & USNV & .034 \\
\hline Red Bird Mine & USNV & 53. \\
\hline Red Cloud Mine & USOR & 2.2 \\
\hline Red Ore Mine & USHV & 58. \\
\hline Red Rock Mine & USNV & 170. \\
\hline Red Rose & USNV & 1.0 \\
\hline
\end{tabular}




\begin{tabular}{|c|c|c|}
\hline Deposit name & Locational & $\begin{array}{l}\text { Mercury content } \\
\text { (Metric tons) }\end{array}$ \\
\hline Reward Property & USNV & 19 \\
\hline Rick Property & USNV & .069 \\
\hline Rimrock and Homestake & USNV & .69 \\
\hline Roba-Westfall & USOR & .41 \\
\hline Rosebud Prospect & USNV & .24 \\
\hline Round Mountain & USOR & .069 \\
\hline Roxana & USOR & .24 \\
\hline Royal Reward & USWA & .14 \\
\hline Sacramento Gold Mine & USUT & 120. \\
\hline San Pedro Group & USNV & 5.3 \\
\hline Senator & USNV & 4.8 \\
\hline Sheebar & USNV & 1.7 \\
\hline Silver Cloud & USNV & 34. \\
\hline Starlight Mine & USNV & 5.6 \\
\hline Steamboat Springs & USNV & 1.0 \\
\hline Steens Mountain Mine & USOR & 1.2 \\
\hline Stockton Property & USNV & .69 \\
\hline Stickland Butte & USOR & .34 \\
\hline Taylor Ranch & USOR & 10. \\
\hline Teapot Prospect & USNV & .14 \\
\hline Tiptop Prospect & USNV & 3.4 \\
\hline Towner & USOR & 6.2 \\
\hline Valentine & USID & .034 \\
\hline Van Ness & USNV & 26. \\
\hline Virginia Claims & USID & .34 \\
\hline Vulture & USNV & .34 \\
\hline Walker Mine & USNV & 3.8 \\
\hline War Eagle & USOR & 23. \\
\hline Washington $\mathrm{Hill}$ & USNV & 1.7 \\
\hline Watson & USOR & .034 \\
\hline White Peaks Group & USNV & 35. \\
\hline Whaley's & USNV & .69 \\
\hline
\end{tabular}




\begin{tabular}{lcl}
\multicolumn{1}{c}{ Deposit name } & Locational & $\begin{array}{l}\text { Mercury content } \\
\text { (Metric tons) }\end{array}$ \\
\hline Wildhorse & USNV & 30. \\
Yellowcat & USNV & .69 \\
a/ See Table 11 for location codes. &
\end{tabular}


The classification of nickel deposits (Naldrett, 1973) has changed rapidly in the last ten years as the result of new information about the geology of these deposits. Thus a set of three empirical criteria were used to obtain a set of data for the RAMRAP nickel deposit model. The criteria used to select data for the intrusive nickel sulfide model were: deposits from orogenic belts only were selected, deposits only with reported nickel and copper grades and an associated tonnage were selected, and deposits suspected of having extrusive ultrabasic host rocks were not selected. 
Table 6.--Intrusive Nickel Sulfide Deposits

Deposit name Locationa/ Ni grade Cu grade Tonnage Reference (Percent) (Percent) (Metric tons

$\left.\times 10^{6}\right)$

\begin{tabular}{|c|c|c|c|c|c|}
\hline Axis Lake & CNSK & .50 & .28 & 3.4 & 2 \\
\hline Bird River & CNMN & .50 & .40 & .30 & 5,4 \\
\hline Carr Boyd & AUWA & 1.41 & .49 & 1.8 & 5 \\
\hline Giant Mascot & CNBC & .65 & .30 & 5.7 & 5,4 \\
\hline Gordon Lake & CNOH & .85 & .29 & 1.7 & 5,4 \\
\hline Kelly Lake & CNQB & .67 & .73 & 1.2 & 2 \\
\hline Kluane Lake & NCYU & 2.04 & 1.42 & .57 & 5,4 \\
\hline Lakemont & CNON & .60 & .40 & 1.8 & 2 \\
\hline Limerick & CNON & .91 & .25 & 1.8 & 5 \\
\hline Lorraine & CNQB & .60 & 1.60 & .50 & 7 \\
\hline Lynn Lake & CNMN & 1.22 & .62 & 14. & 7,3 \\
\hline Macassa & CNON & .70 & .25 & 3.5 & 2 \\
\hline Maskwa & CNMN & 1.06 & .34 & 1.3 & 5,6 \\
\hline Midrim & CNQB & .46 & .70 & .39 & 2 \\
\hline Nembeiben Lake & CNSK & .36 & .40 & 5.6 & 2 \\
\hline Norpax & CNON & .85 & .35 & 1.7 & 4,2 \\
\hline Pickle Crow & CNON & .20 & 1.70 & 12. & 5,6 \\
\hline Populus Lake & CNON & 1.06 & .54 & 6.3 & $7,2,4$ \\
\hline Renzy Lake & CNQB & .70 & .70 & 1.2 & 2 \\
\hline St. Fabien & CNQB & .37 & 1.08 & 1.2 & 2 \\
\hline St. Stephen & CNNB & .97 & .52 & 2.3 & 7,2 \\
\hline Temagami Mine & CNON & .47 & 1.04 & .70 & 2 \\
\hline ZuTapa & CNQB & .55 & .48 & 1.5 & 2 \\
\hline Belleterre & CNQB & .67 & .73 & 2.0 & 7 \\
\hline Canalask & CNYU & 1.50 & .04 & .50 & 2 \\
\hline Juneau lake & CNON & .87 & .59 & 2.0 & 2 \\
\hline Retty Lake & CNQB & .55 & .95 & 1.4 & 2 \\
\hline Chance Lake & CNQB & .89 & .66 & .65 & 2 \\
\hline Gagné & CNQB & .88 & .44 & .27 & 2 \\
\hline
\end{tabular}


Deposit name Locational Nigrade Cugrade Tonnage Reference (Percent) (Percent) (Metric tons

\begin{tabular}{llllll}
\hline Nicobi Lake & CNQB & .48 & .25 & 1.6 & 2 \\
Sudbury Shakespeare & CNON & .34 & .40 & 2.7 & 2 \\
HcVittie - Graham & CNON & .63 & .38 & 2.3 & 2 \\
Renner Prospect & CNON & .29 & .26 & 2.6 & 2 \\
Nicopar & CNON & .44 & .19 & .34 & 2 \\
Jacobus Prospect & CNON & .41 & .42 & .85 & 2 \\
Diadem Prospect & CNON & .18 & .50 & .45 & 2 \\
Cat Lake & CNMN & .24 & .58 & .59 & 2 \\
Ivy Group & CNSK & .29 & .08 & 3.9 & 2 \\
E \& L & CNBC & .80 & .62 & 3.0 & 2 \\
Cons Mogador & CNQB & .82 & .68 & .32 & 2 \\
Dumont (Wendel1) & CNQB & .68 & .30 & .06 & 2 \\
R. H. Nickel & CNQB & .51 & .74 & .09 & 2 \\
Nattagami Lake & CNQB & .39 & .28 & .50 & 2 \\
Owens & CNON & .53 & .27 & .09 & 2 \\
New Dominion & CNON & .90 & .75 & .04 & 2 \\
Keevil, etc. & CNMN & .60 & 1.00 & 2.3 & 2 \\
Ore Fault & CNMN & .48 & .20 & 1.5 & 2 \\
Montcalm Township & CNON & 1.00 & .35 & 2.7 & 2
\end{tabular}

a/ See Table 11 for Tocation codes. 


\section{Vein Gold}

The definition of what constitutes a deposit is perhaps most difficult for vein deposits. Often several mines operate in the same vein or a mine may operate in several veins. Thus, several mines may be combined to represent one deposit. As with mercury deposits, data on the contained metal of the deposits, rather than on the grades and tonnages, is generally reported. 
Table 7.--Vein Gold Deposits

\begin{tabular}{|c|c|c|c|}
\hline Deposit name & Locationa/ & $\begin{array}{l}\text { Gold content } \\
\text { (Metric tons) }\end{array}$ & Reference. \\
\hline Aurum Deposit & CNBC & 11. & 1 \\
\hline Artic Mine & CNYU & 5.3 & 5 \\
\hline Big Missouri & CHBC & 1.8 & 3 \\
\hline Bralorne-Pioneer & CNBC & 120. & 3 \\
\hline Brown McDade & CNYU & .61 & 1 \\
\hline SK \#3 Vein & CNBC & .055 & 1 \\
\hline Cariboo Gold Quartz - Island Mtn. & CNBC & 64. & 3 \\
\hline Cariboo-Mckinney & CNBC & 2.55 & 3 \\
\hline $\begin{array}{l}\text { Zeballos-Spud Valley-Privateer } \\
\text { Mount Zeballos-Central Zeballos }\end{array}$ & CNBC & 12. & 4 \\
\hline Detonia & CNBC & 1.2 & 3 \\
\hline Engineer & CNBC & .56 & 2 \\
\hline Granite Poorman & CNBC & 2.0 & 3 \\
\hline Hi Do & CNBC & .62 & 1 \\
\hline Laforma &. CNYU & 1.84 & 1,5 \\
\hline Le Roi-Centre Star-War Eagle & CNBC & 84 & 3 \\
\hline Lucky Seven & CNBC & .025 & 1 \\
\hline Mt. Nansen & CNYU & 2.1 & 1 \\
\hline Polaris Taku & CNBC & 7.2 & 3 \\
\hline Premier & CNBC & 56. & 3 \\
\hline Salmon Gold & CNBC & 3.4 & 1 \\
\hline $\begin{array}{l}\text { Sheep Creek-Gold Belt, Kootenay } \\
\text { Bell-Reno }\end{array}$ & CNBC & 23 & 3 \\
\hline Arlington & CNBC & 3.09 & 4 \\
\hline Erie Creek & CNBC & 2.9 & 4 \\
\hline Roche Deboule Mine & CNBC & .13 & 4 \\
\hline Smith Nash & CNBC & 3.3 & 1 \\
\hline Surf Inlet & CNBC & 12. & 3 \\
\hline Union Franklin & CNBC & 1.71 & 2 \\
\hline Velvet & CNBC & .62 & 3 \\
\hline Venus Mine & CNYU & 2.3 & 1 \\
\hline
\end{tabular}




\begin{tabular}{llll}
\hline Vidette & CNBC & 1.2 & 1,3 \\
Ymir & CNBC & 3.4 & 3 \\
Alaska Juneau & USAK & 76. & 4 \\
Berness Bay & USAK & 2.0 & 4 \\
Early Bird Mine & CNBC & 1.5 & 4 \\
Fairview-0liver & CNBC & .48 & 4 \\
Nighthawk & USWA & 1.4 & 4 \\
Perseverance & USAK & 13. & 4 \\
Republic & USWA & 24. & 4 \\
Slate Creek & USWA & .83 & 4 \\
Surf Point & CNBC & 11. & 4 \\
Treadweit & USAK & 95. & 4 \\
Wenatchee & USWA & 5.4 & 4 \\
Wingdam Mine & CNBC & 1.5 & 4
\end{tabular}

a/ See Table 11 for location codes. 


\section{Mafic Volcanogenic Copper}

Deposits were selected on the basis of the presence of massive and irregular sulfide bodies in or closely associated with mafic volcanic rocks in eugeosynclines. Sulfide minerals include pyrite, chalcopyrite, and, for many deposits, sphalerite. The mafic volcanic rocks generally present were basalt or other basic lavas; deposits having any associated felsic or intermediate volcanic rocks were excluded. A copper grade and associated tonnage were required in order for the deposit to be included in the data used. 
Table 8.--Mafic Volcanogenic Copper Deposits

\begin{tabular}{|c|c|c|c|c|c|}
\hline Deposits name & Locationa/ & $\begin{array}{l}\text { Copper } \\
\text { grade } \\
\text { (Percent) }\end{array}$ & $\begin{array}{l}\text { Zinc } \\
\text { grade } \\
\text { (Percent) }\end{array}$ & $\begin{array}{l}\text { Tonnage } \\
\text { (Metric } \\
\text { tons } \\
\times 10^{5} \text { ) } \\
\end{array}$ & Reference \\
\hline Besshi & JPAN & 3.80 & & 190. & 1 \\
\hline Sazare & JPAN & 2.30 & & 1.6 & 1 \\
\hline Surigad $\mathrm{Cu}-\mathrm{Zn}$ & PLPN & 2.50 & 1.00 & 1.0 & 1 \\
\hline Surigad $\mathrm{Cu}$ & PLPN & 1.40 & & 228. & 1 \\
\hline Mindanao & PLPN & 4.10 & & 4.37 & 1 \\
\hline Sankarapi & INDS & 1.80 & & 100 & 1 \\
\hline Balabae Is. & PLPN & 4.70 & & 4.75 & 1 \\
\hline Slovinky & $C Z S L$ & .80 & & 81.3 & 1 \\
\hline Skouries & CRCE & .70 & & 222. & 1 \\
\hline Caracassi & GRCE & .80 & .60 & 19.4 & 1 \\
\hline Limogardi & GRCE & 1.50 & & 29.3 & 1 \\
\hline Kure & TRKY & 2.10 & .70 & 43.1 & 1 \\
\hline Asikoy-Kurewest & TRKY & 1.90 & & 16. & 1 \\
\hline Gedak & TRKY & 5.00 & & 1.0 & 1 \\
\hline Apliki & CPRS & 1.80 & .60 & 16.5 & 1 \\
\hline Kalavassos & CPRS & 1.50 & .70 & 24.7 & 1 \\
\hline Kynousa & CPRS & 2.30 & 3.40 & 5.15 & 1 \\
\hline Limnt & CPRS & 1.40 & & 59.8 & 1 \\
\hline Mauri Sykia & CPRS & 2.00 & & 3.5 & 1 \\
\hline Maurououni & CPRS & 4.00 & .40 & 150 & 1 \\
\hline Petka & CPRS & 1.80 & 1.50 & 5.0 & 1 \\
\hline Skouriotissa & CPRS & 2.30 & & 66.8 & 1 \\
\hline Twin J-MT & CNBC & 2.80 & .86 & 2.3 & 1 \\
\hline Campbell Chibougamau & CNQB & 3.00 & & 134. & 1 \\
\hline Perill Islm & CNQB & 2.00 & & 12.5 & 1 \\
\hline Chibougamau Explores & CNQB & .90 & & 5.4 & 1 \\
\hline Outokiepu & FNLD & 4.00 & .80 & 200. & 1 \\
\hline Ropklev & NRWG & 2.60 & 5.00 & 2.34 & 1 \\
\hline Vignas & NRWG & 3.20 & 3.50 & 8.44 & 1 \\
\hline Lokken & NRWG & 2.60 & 9.80 & 183 & 1 \\
\hline
\end{tabular}




\begin{tabular}{lccccc} 
Deposit name & Locationa/ & $\begin{array}{l}\text { Copper } \\
\text { grade } \\
\text { '(Percent) }\end{array}$ & $\begin{array}{l}\text { Zinc } \\
\text { grade } \\
\text { (Percent) }\end{array}$ & $\begin{array}{l}\text { Tonnage Reference } \\
\text { (Metric } \\
\text { tons } \\
\left.\times 10^{5}\right)\end{array}$ \\
\hline Fordal & NRWG & 2.80 & 1.20 & 88.2 & 1 \\
Roroscu & NRWG & 1.70 & .07 & 67. & 1 \\
Killingdal & NRWG & 1.80 & .66 & 9.1 & 1 \\
Gjersvik & NRWG & 2.10 & & 14.3 & 1 \\
Tuerrejellet & NRWG & 2.50 & 1.50 & 8.0 & 1 \\
Stekenjokk & SWDN & 1.60 & 3.50 & 320. & 1 \\
Sain Bell-Chessy & FRNC & 5.0 & 6.00 & 400. & 1
\end{tabular}

a/ See Table 11 for location codes. 
Felsic to Intermediate Volcanogenic Sulfide

Deposits were classified in this group if they consisted of massive and irregular sulfide bodies in or closely associated with felsic to intermediate volcanic rocks in submarine flysch environments. The sulfide minerals consisted of chalcopyrite, pyrite or pyrrhotite, sphalerite, and/or galena. In order to be included in the data set, both a copper grade and associated tonnage had to have been reported. For some deposits gold and/or silver grades and associated tonnages were reported. Many of the reported tonnages associated with gold and silver grades were significantly lower than the tonnages associated with copper grades in the same deposits. It was assumed that for many deposits only a portion of the deposit had goid and silver grades high enough to be reported and that it would therefore be misleading to present these grades with the higher tonnages associated with the copper grades. Thus, for silver and gold only contained metal is reported. Six deposits from Newfoundland that, according to the information available, met the criteria for this deposit type have been identified by D. Cox (personal communication, 1979) as belonging to the mafic volcanogenic deposit type and are not used in the revised grade-tonnage model for felsic to intermediate volcanogenic deposits. 
Table 9.--Felsic to Intermediate Volcanogenic Deposits

\begin{tabular}{|c|c|c|c|c|c|c|c|c|}
\hline Deposit namea/ & $\begin{array}{l}\text { Loca- } \\
\text { tionb/ }\end{array}$ & $\begin{array}{l}\text { Gold } \\
\text { ton- } \\
\text { nage } \\
\text { (Metric } \\
\text { tons) }\end{array}$ & $\begin{array}{l}\text { Silver } \\
\text { ton- } \\
\text { nage } \\
\text { (Metric } \\
\text { tons) }\end{array}$ & $\begin{array}{l}\text { Copper } \\
\text { grade } \\
\text { (Per- } \\
\text { cent) }\end{array}$ & $\begin{array}{l}\text { Zinc } \\
\text { grade } \\
\text { (Per- } \\
\text { cent) }\end{array}$ & $\begin{array}{l}\text { Lead } \\
\text { grade } \\
\text { (Per- } \\
\text { cent) }\end{array}$ & $\begin{array}{l}\text { Tonnage } \\
\text { (Metric } \\
\text { tons } \\
\times 10^{4} \text { ) }\end{array}$ & $\begin{array}{l}\text { Ref- } \\
\text { er- } \\
\text { ences }\end{array}$ \\
\hline Suffield Mine & CNQB & .50 & 70 . & 1.28 & 6.45 & .60 & 100 & 1 \\
\hline Solbec Mine & CNQB & .60 & 51. & 1.39 & 4.80 & .74 & 178. & 1 \\
\hline Cupra Mine & CNQB & .503 & 36.7 & 3.77 & 3.70 & 1.20 & 99.4 & 1 \\
\hline No. 6 Mine & CNQB & & 799. & .45 & 5.63 & 2.25 & 1480 & 1 \\
\hline No. 12 Mine-Anaconda & CNNB & & 1920. & .27 & 8.90 & 3.54 & 5090 & 1 \\
\hline Anaconda Orebody & CNNB & & 165. & .20 & 7.43 & 3.03 & 182. & 1 \\
\hline Heath Steeie Orebody & CNNB & 4.07 & 542. & 1.10 & 7.10 & 2.90 & 775 . & 1 \\
\hline Captain Mines property & CNNB & .02 & 2.7 & 1.99 & & & 57.6 & 1 \\
\hline Nigadoo River & CNNB & & 171. & .34 & 2.80 & 3.00 & 139. & 1 \\
\hline Big Hill & CNNB & & 109. & .14 & 1.20 & .80 & 80.4 & 1 \\
\hline Banet Prospect & USME & 2.5 & 6.5 & 1.75 & & & 38.5 & 1 \\
\hline Mindamar & USME & & 32.7 & .66 & 5.90 & 1.35 & 83.3 & 1. \\
\hline York Harbor & CNNS & & & 2.63 & 8.25 & & 21.8 & 1 \\
\hline Rambier Mine Property* & CNNF & 7.3 & 3.9 & 1.43 & 1.74 & & 247. & 1 \\
\hline Tilt Cove Cu Pyrites* & CNNF & 9.8 & 11.5 & 1.47 & & & 1220 & 1 \\
\hline Tilt Cove Chalco* & CNNF & 6.8 & 4.5 & 5.50 & & & 151. & 1 \\
\hline Betts Cove* & CNNF & 1.2 & 3.5 & 10.00 & 2.00 & & 13. & 1 \\
\hline Whalesback* & CNNF & & & 1.50 & & & 400 & 1 \\
\hline Gull Pond-Gullbridge M. & ${ }^{*} \mathrm{CNNF}$ & & & 1.47 & & & 367. & 1 \\
\hline Avoca $\mathrm{Cu}$ & IRLD & & & 1.14 & 1.12 & .08 & 2050 & 1 \\
\hline Vanze Mine & CNQB & & & 5.20 & 3.71 & & 20. & 1 \\
\hline Morbrun Property & CNQQB & 4.5 & 53.4 & .69 & 2.20 & & 304. & 1 \\
\hline Golden Manitou Mine & CNQB & 6.0 & 204. & .97 & 3.20 & & 921. & 1 \\
\hline Zulaka Mine & CNQB & & & .48 & & & 169. & 1 \\
\hline Normetal Mine & CNQB & 4.32 & 405. & 1.94 & 7.70 & & 1090 & 1 \\
\hline Kelly-Osmond Property & CNQB & & 28.3 & .73 & 6.90 & & 100 & 1 \\
\hline Joutel Copper Mine & CNQB & & & 2.05 & 1.30 & & 160. & 1 \\
\hline Orchan Mine & CNQB & 1.3 & 106. & 1.26 & $11: 00$ & & 327. & 1 \\
\hline New Hosco Mine & CNQB & & & 2.64 & .14 & & 174. & 1 \\
\hline
\end{tabular}


Deposit namea/

Loca- Gold

tionb/ ton-

nage

(Metric

tons)

\begin{tabular}{|c|c|c|c|c|c|c|c|}
\hline Norita Mine & CNQB & & & .64 & 7.30 & & 80. \\
\hline Garon Lake Property & CNQB & & & 2.12 & & & 29.5 \\
\hline Besakoa & MLGS & & & .60 & & & 77.7 \\
\hline Saxbergst & SWDN & & & .90 & 4.50 & 3.00 & 200. \\
\hline Garkenberg & SWDN & & & .30 & 5.20 & & 1000 \\
\hline Rakkejaur & SWDN & 12. & 450. & .20 & 2.60 & & 1000 \\
\hline Pynasalmi & FNLD & .80 & 63.8 & .85 & 2.80 & .06 & 375. \\
\hline Ely Mine & USVT & & & 3.50 & & & 50 \\
\hline Elizabeth Mine & USVT & & - & 1.80 & & & 639. \\
\hline Huntingdon Mine & CNQB & .07 & & 2.00 & & & 48.9 \\
\hline Harvey Hili Mine & CNQB & & & 1.85 & & & 76.4 \\
\hline Quandt & CNSK & & & 2.75 & 1.50 & & 50. \\
\hline Flin Flon Orebody & CNMN & 119 . & 1700 . & 2.99 & 4.40 & & 5690. \\
\hline Mandy Mine & CNMN & .40 & 6.8 & 5.47 & 16.50 & & 21. \\
\hline Schist Lake & CNMN & 6.9 & 16.8 & 5.58 & 6.60 & & 91.2 \\
\hline Flexar & CNMN & .20 & .9 & 4.11 & .40 & & 24.1 \\
\hline Sourdough Bay & CNSK & & & 3.00 & & & 75. \\
\hline Vamp Lake & CNSK & 1.01 & 6.8 & 1.10 & 1.60 & & 50. \\
\hline Morton L-Dickson L & CNMN & & & 2.53 & 3.20 & & 57.7 \\
\hline Fox Lake & CNMN & & & 1.74 & 2.35 & & 122. \\
\hline Willecho Property & CNON & & 97.1 & .77 & 3.10 & .01 & 243. \\
\hline Kotia-Kamiskotia Mine & CNON & & & 1.69 & 2.00 & & 625. \\
\hline Canadian Jamieson $\mathrm{Pr}$. & $\mathrm{CNON}$ & & & 2.90 & 4.80 & & 51.9 \\
\hline Horne Mine & CNQB & 257. & & 2.11 & & & 5900. \\
\hline Waite Dufault Property & CNQB & & & 1.50 & & & 25.2 \\
\hline West Wasa Property & CNQB & & & 2.00 & & & 154. \\
\hline Munaz Property & CNQB & 11.2 & 74.9 & 1.17 & 2.40 & & 308. \\
\hline Lake Dufault Mines & CNQB & 2. & 118. & 3.30 & 6.80 & & 257. \\
\hline Waite Amulet Mine & CNQB & 11.2 & 354. & 4.71 & 3.10 & & 955. \\
\hline Cerro Roja Orebody & SPAN & 32.7 & 792. & .80 & & & 4860. \\
\hline Santa Domingo & PORT & & & 1.17 & & & 2150. \\
\hline Aznal Collar & SPAN & & & 2.20 & & & 500 \\
\hline
\end{tabular}




\begin{tabular}{|c|c|c|c|c|c|c|c|c|}
\hline Deposit namea/ & $\begin{array}{l}\text { Loca- } \\
\text { tionb/ }\end{array}$ & $\begin{array}{l}\text { Gold } \\
\text { ton- } \\
\text { nage } \\
\text { (Metric } \\
\text { tons) }\end{array}$ & $\begin{array}{l}\text { Silver } \\
\text { ton- } \\
\text { nage } \\
\text { (Metric } \\
\text { tons) }\end{array}$ & $\begin{array}{l}\text { Copper } \\
\text { grade } \\
\text { (Per- } \\
\text { cent) }\end{array}$ & $\begin{array}{l}\text { Zinc } \\
\text { grade } \\
\text { (Per- } \\
\text { cent) }\end{array}$ & $\begin{array}{l}\text { Lead } \\
\text { grade } \\
\text { (Per- } \\
\text { cent) }\end{array}$ & $\begin{array}{l}\text { Tonnage } \\
\text { (Metric } \\
\text { tons } \\
\times 10^{4} \text { ) }\end{array}$ & $\begin{array}{l}\text { Ref- } \\
\text { er- } \\
\text { ences }\end{array}$ \\
\hline Porvenir $\mathrm{Cu}$ & SPAN & & & 10.00 & & & 2. & 1 \\
\hline Zyuzelski & URRS & & & 4.20 & & & 143. & 1 . \\
\hline Salair & URRS & & & .90 & 8.80 & .90 & 1270. & 1 \\
\hline Burraga & AUNW & & & 3.70 & & & 50.3 & 1 \\
\hline Read Roseberry & AUTS & 16.3 & 1840 & .95 & 20.00 & 6.00 & 1070. & 1 \\
\hline Anyox Hidden Creek & CNBC & 3.4 & 187. & 1.28 & & & 2390 & 1 \\
\hline Britannia Mine & CNBC & 11.4 & 133. & .95 & .16 & .03 & 4420 & 1 \\
\hline Iron Mt. Mine & USCA & 7.0 & 520. & 3.35 & 3.50 & & 873. & 1 \\
\hline Mammoth Mine & USCA & 3.4 & 202. & 3.95 & 4.60 & & 309. & 1 \\
\hline United Verde Ext. Mine & UZAZ & 4.3 & 185. & 9.23 & & & 389. & 1 \\
\hline Iron King Mine & UZAZ & 12.2 & 524. & 1.73 & 6.65 & 2.30 & 500. & 1 \\
\hline Macuchi Mine & ECDR & 4.61 & 5.2 & 5.00 & & & 62.4 & 1 \\
\hline Aquire & CILE & & & 2.00 & & & 340. & 1 \\
\hline LaBallade Mine & NCLD & & & 12.50 & & & 8.64 & 1 \\
\hline Meretrice Mine & NCLD & & & 4.00 & $29.00:$ & 25.00 & 9.5 & 1 \\
\hline Herin & ITLY & & & 2.50 & & & 15. & 1 \\
\hline Fabriche & ITLY & & & 5.00 & & & 3.0 & 1 \\
\hline Knappenstube & ASTR & & & 1.50 & & & 1000 & 1 \\
\hline Lahanos & TRKY & & & 2.00 & & & 12.5 & 1 \\
\hline Israil & TRKY & & & 2.55 & & & 25.8 & 1 \\
\hline Girlak-Gorele & TRKY & & & 2.00 & & & 250. & 1 \\
\hline Dzansul & URGR & & & 3.00 & & & 360. & 1 \\
\hline Tchorokh & URGR & & & 4.50 & & & 50. & 1 \\
\hline Myra-Falls Lynx & CNBC & 10.9 & 623. & 8.30 & 1.30 & & 471. & 1,2 \\
\hline Kidd Creek Mine & CNON & & 15600. & 2.43 & 7.00 & .20 & 15700. & 1,2 \\
\hline White Lake & CNMN & .23 & 1.6 & 2.16 & 5.70 & .40 & 44.1 & 1,2 \\
\hline Vendome & CNQB & 1.23 & 57.1 & .52 & 6.70 & .30 & 112. & 1,2 \\
\hline Geco Mine & CNON & & 1510 & 1.94 & 3.90 & & 4720. & 1,2 \\
\hline Matsuki & JPAN & & & 1.00 & 3.00 & 2.00 & 922. & 2 \\
\hline Shansanai & JPAN & & & 1.49 & 2.22 & .56 & 1030. & 2 \\
\hline Mattagami Mine & CNBQ & 9.08 & 45. & .55 & 6.76 & & 2670. & 1,2 \\
\hline
\end{tabular}




\section{Copper Skarn}

Deposits were classified as copper skarns if they included disseminated chalcopyrite and, in most cases, magnetite in skarns. Typically, the deposits occur in metasomatized limestones in contact with younger igneous rocks. Although many of the deposits have gold and silver grades reported, the reported values are in some cases inconsistent; therefore gold and silver grades are not provided here. It is possible that some - of the values for individual deposits reported here may actually represent several deposits in a district. Three deposits used in the gradetonnage model developed for the RAMRAP reports have been found to be duplicates and have been removed from this list; seven other deposits were added to the list. 
Table 10.--Copper Skarn Deposits

\begin{tabular}{|c|c|c|c|c|}
\hline Deposit name & Location a/ & $\begin{array}{l}\text { Grade } \\
\text { (Percent) }\end{array}$ & $\begin{array}{l}\text { Tonnage } \\
\text { (metric } \\
\text { tons } \\
\times 10^{4} \\
\end{array}$ & Reference \\
\hline Gaspe Copper-Murdochville & CNQB & 1.08 & 91.4 & 2 \\
\hline Wexford Mines Prop. & CHBC & 1.52 & 10. & 2 \\
\hline Tasu-Wesfrob Mine $\mathrm{Cu}$ & CNBC & .66 & 15.2 & 2 \\
\hline Yreka & CNBC & 3.60 & .21 & 2 \\
\hline Benson Lake & CNBC & 1.60 & 1.26 & $2 \cdot$ \\
\hline Kasaan Peninsula & USAK & 2.13 & 1.89 & 2 \\
\hline Craigmont $\mathrm{Cu}$ & CNBC & 1.84 & 27.9 & 2 \\
\hline Phoenix & CNBC & .80 & 2500. & 2 \\
\hline Snowshow N. Phoenix & CNBC & 1.05 & 60. & 2 \\
\hline Meme Zone & HATI & 1.70 & 207 & 2 \\
\hline Cassius Prop. & HATI & .65 & 700. & 2 \\
\hline Cerro deCobte-Cundina Marca & CLBA & 3.00 & 33.3 & 2 \\
\hline Cobriza & PERU & 2.53 & 710. & 2 \\
\hline Tintaya & PERU & 3.00 & 700 & 2 \\
\hline Chal cobamba & PERU & 1.50 & 3500 . & 2 \\
\hline Kamaish $\mathrm{Cu}-\mathrm{Ag}-\mathrm{Au}$ & JPAN & 3.93 & 10. & 2 \\
\hline Agdrdo and Brosso & ITLY & 1.70 & 640. & 2 \\
\hline Traversella & ITLY & 2.00 & 40 & 2 \\
\hline Sasca Montana-Neu. Moldova & RMNA & 2.50 & 100. & 2 \\
\hline Malko Trnova & BULG & 1.76 & 196. & 2 \\
\hline Kedabeg Cu & URAZ & 3.50 & 170. & 2 \\
\hline Loei-Chiengkarn Area Cu & THLD & .90 & 8000 & 2 \\
\hline Mackey District & USID & 5.37 & 46.9 & 2 \\
\hline Standzha District & BULG & 1.75 & 1000 . & 2 \\
\hline Cornell : & CNBC & 3.30 & 4.08 & 2 \\
\hline Marble Bay & CNBC & 2.39 & 28.5 & 1 \\
\hline Vananda & CNBC & 1.43 & 6.35 & 1 \\
\hline Indian Chief & CNBC & 1.59 & 142.6 & 3 \\
\hline Blue Grouse & CNBC & 2.73 & 24.9 & 1 \\
\hline
\end{tabular}




\begin{tabular}{lcclc} 
Deposit name & Location a/ & $\begin{array}{l}\text { Grade } \\
\text { (Percent) }\end{array}$ & $\begin{array}{l}\text { Tonnage } \\
\text { (Metric } \\
\text { tons } \\
\times 10^{4}\end{array}$ & Reference \\
\hline Mother Lode & CNBC & .89 & 392 & 1 \\
Sunset & CNBC & .83 & 18. & 3 \\
B.C. & CNBC & 4.37 & 9.34 & 1 \\
Emma & CNBC & .98 & 23.2 & 1 \\
Oro Denoro & CNBC & 1.00 & 118.2 & 3 \\
Queen Victoria & CNBC & 2.04 & 4.44 & 1 \\
Carr Fork & USUT & 1.84 & 5550. & 5 \\
Copper Canyon & USNV & .80 & 1590. & 4 \\
Johnson Camp & USAZ & .85 & 2000. & 5 \\
Marbie Peak & USAZ & 2.28 & 3610. & 5 \\
Pima-Mission & USAZ & .56 & 37800. & 5 \\
Miami East & USAZ & 1.95 & 5000. & 5 \\
Pinos Altos & USNM & 2.0 & 700. & 5 \\
a/ See Table 11 for location & Codes & & & it
\end{tabular}


Table 11.--Location Codes

ASTR Austria

AUNW Australia, New South Wales

AUTS

AUWA

BRSL Brazil

BULG Bulgaria

CILE Chile

CLBA Colombia

CNBC Canada, British Columbia

CNMN

CNNB

CNNF

CNNS

CNNT

CNON

CNQB

CNSK

CNYU

CPRS Cyprus

CZSL Czechoslovakia

ECDR Ecuador

FNLD Finland

FRNC France

GRCE Greece

GRLD Greenland

HATI Haiti

INDS Indonesia

IRLD Ireland

ITLY Italy

JPAN Japan
KORA Korea

MLGS Malegasy Republic

NCLD New Caledonia

NRWG Norway

PERU Peru

PLPN Philippines

PORT Portugal

RMNA Rumania

SPAN Spain

SWDN Sweden

THLD Thailand

TRKY Turkey

URAZ U.S.S.R., Azerbaidzhan

URGR " ., Georgia

URRS " , Russian Republic

URTD " , Turkmenia

USAK U.S.A., Alaska

USAZ ", Arizona

USCA ", California

USCO ", Colorado

USID ", Idaho

USME ", Maine

USMT ", Montana

USNM ", New Mexico

USNV ", Nevada

USOR ", Oregon

USUT ", Utah

USVT ", Vermont

USWA ", Washington

VNZL Venezuela 
Table 12.--Grade and Tonnage Models

(metric units)

NS, not significant; *, significant at 5-percent level; **, significant at 1-percent level

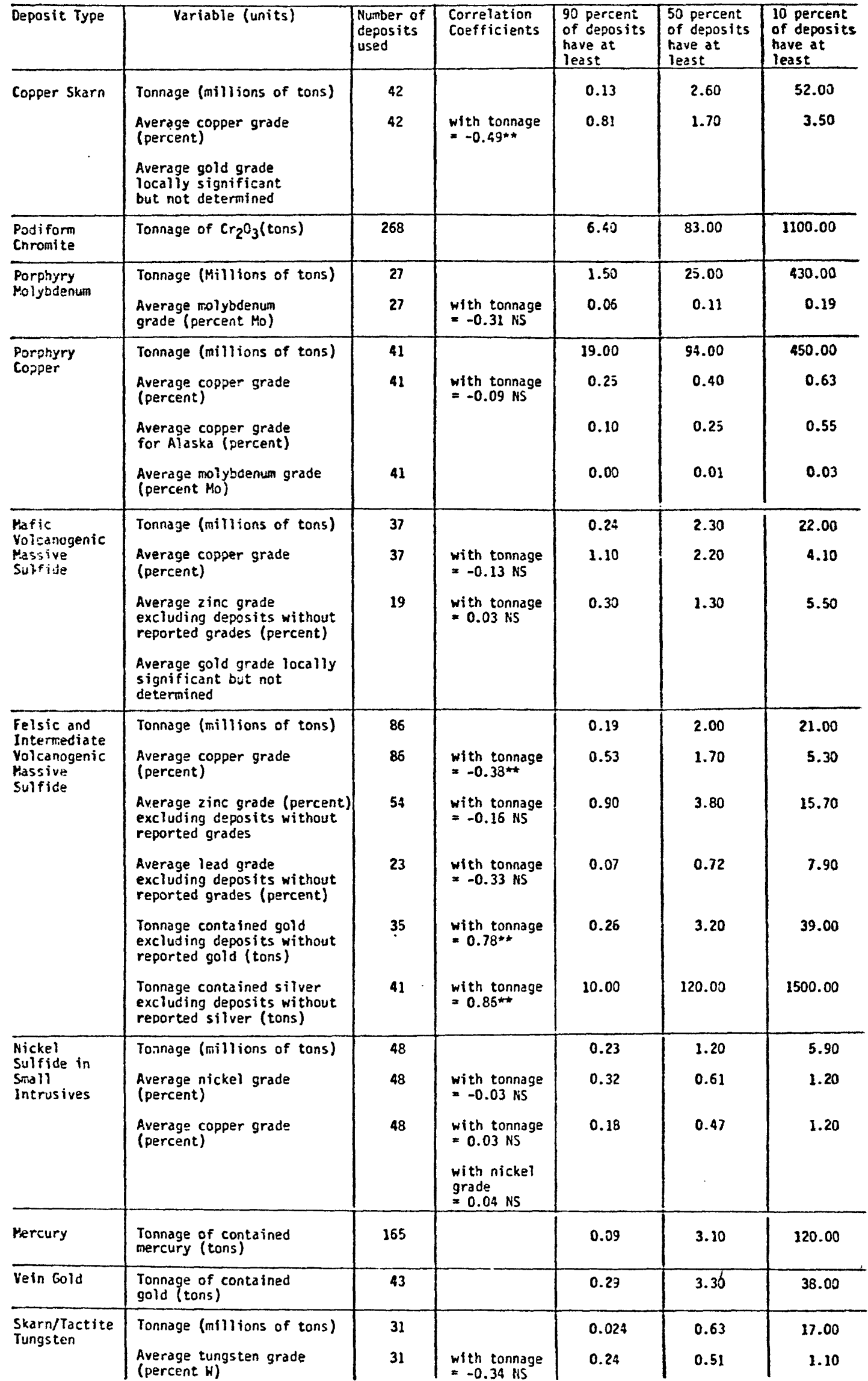




\section{REFERENCES}

\section{Chromite}

California Division of Mines, 1946-1965, Geological investigations of chromite in California: California Div. Mines Bul1. 134, published by chapers as follows:

Part I. Klamath Mountains

Chap. I. Del Norte County, by F. G. Wells, F. W. Cater, Jr., and G. A. Rynearson, 1946, p. 1-76.

Chap. II. Siskiyou County, By F. G. Wells and F. W. Cater, Jr., 1950 , p. 77-127.

Chap. III. Shasta, Tehama, Trinity, and Humboldt Counties, by F. G. Wells and H. E. Hawkes, 1966.

Part II. Coast Ranges

Chap. I. Northern Coast Ranges, by D. H. Dow and T. P. Thayer, 1946 , p. 1-38.

Chap. II. Southern Coast Ranges, by G. W. Walker and A. B. Griggs, 1953 , p. 39-88.

Part III. Sierra Nevada

Chap. I. Tuolumne and Mariposa Counties, by F. W. Cater, Jr., 1948, p. 1-32.

Chap. II. Calaveras and Amador Counties, by F. H. Cater, Jr., 1948, p. 33-60.

Chap. III. Tulare and eastern Fresno Counties, by G. A. Rynearson, 1948, p. 61-104.

Chap. IV. EI Dorada County, by F. W. Cater, G. A. Rynearson, and D. H. Dow, 1951, p. 105-167.

Chap. V. Northern Sierra Nevada (Placer, Nevada, Sierra, Yuba, Butte, and Plumas Counties) By G. A. Rynearson, 1953, p. 169-323. 


\section{Porphyry Copper}

(1) Annis, R.C., Cranstone, D.A., and Vallée, M., 1976, A Survey of Known Mineral Deposits in Canada That Are Not Being Mined, Canadian Dept. of Energy, Mines and Resources, Ottawa, (96) p. Includes Addendum dated 27 May 1977.

(2) Drolet, Jean-Paul, The Demands and Limitations Governing the Mineral Production of Western Canada to 1990, 13 0ct. 1976, Vancouver.

(3) Drummond, A.D., and Godwin, C.I., 1976, "Hypogene Mineralization: An Empirical Evaluation of Alteration Zoning," in Brown, A.S., ed., Porphyry Deposits of the Canadian Cordiliera, Can. Inst. Mining and Metal., Spec. Vol. 15, p. 52-63.

(4) Mining Magazine, 1972, Progress at Similkameen, v. 126, no. 3, (Mar), p. 167.

(5) Pilcher, S.H., and McDougal1, J.J., 1976, "Characteristics of Some Canadian Cordilleran Porphyry Prospects," in Brown, A.S., ed., Porphyry Deposits of the Canadian Cordiliera, Can. Inst. Mining and Metal., Spec. Vol. 15, p. 79-82.

(6) Richter, D.H., Singer, D.A., and Cox, D.P., 1975, Mineral Resources Map of the Nabesna Quadrangle, Alaska: U.S. Geological Survey Misc. Field Studies Map MF - 655K, 1 sheet, scale 1:250,000.

(7) Western Miner, 1973, "The Afton Discovery," v. 46, no. 2, Feb., p.36.

(8) Industry sources.

(9) World Mining, 1978, What's Going on in World Mining, v. 31 , no. 12 , p. 88.

(10) Mining Jour., 1979, Major Canadian Copper Deposit, 17 Aug. 1979, v. 293 , no. 7513, p. 126.

(11) Mining Jour., 1979, Canadian Copper-Moly Discovery, 14 Sept. 1979, v. 293 , no. 7517, p. 217. 
Porphyry Molybdenum

(1) American Metals Market, 1980, Quartz Hill's Holy Reserve Upgraded, 27 Feb. 1980, vol. 88 , no. 39, p. 7.

(2) Annis, R.C., Cranstone, D.A., and Vallee, M., 1976, A Survey of Known Mineral Deposits in Canada That Are Not Being Mined, Canadian Dept. of Energy, Mines and Resources, Ottawa, (96) p. Includes Addemdum dated 27 May 1977.

(3) Drummond, A.D., and Godwin, C.I., 1976, "Hypogene Mineralization: An Empirical Evaluation of Alteration Zoning," in Brown, A.S., ed., Porphyry Deposits of the Canadian Cordillera, Can. Inst. Mining and Metal., Spec. vol. 15, p. 52-63.

(4) King, R.U., Shawe, D.R., and MacKevett, E.M., Jr., 1973, "Molybdenum," in Brobst, D.A., and Pratt, W.P., editors, United States Mineral Resources, U.S. Geol. Survey Prof. Paper 820, p. 425-435.

(5) Mining Engineering, 1980, US and International Mineral News Briefs, Jan. 1980 , vol. 32 , no. 1 , p. 17.

(6) Pilcher, S.H. and McDougall, J.J., 1976, "Characteristics of Some Canadian Cordilleran Porphyry Prospects," in Brown, A.S., ,ed., Porphyry Deposits of the Canadian Cordillera, Can. Inst. Mining and Metal., Spec. vol. 15, p. 79-82.

(7) Sutulov, Alexander, 1976, Molybdenum and Rhenium, 1778-1977, University of Concepcion, Chile, $257 \mathrm{p}$.

(8) Sutulov, Alexander, 1978, International Molybdenum Encyclopedia, 1778-1978, vol. 1, Resources and Production: Intermet Publications, Santiago, Chile, 402 p.

(9) Theodore, T.G., 1979, Personal communication.

(10) World Mining, 1979, Six Exploration Diamond Drills at Mount Emmons, June 1977, vol. 32, no. 6, p. 101.

(11) World Mining, 1980, Moly in British Columbia, Feb. 1980, vol. 33, no. 2, p. 65 . 


\section{Tungsten}

(1) Annis, R.C., Cranstone, D.A., and Vallèe, M., 1976, A Survey of Known Mineral Deposits in Canada That Are Not Being Mined, Canadian Dept. of Energy, Mines and Resources, Ottawa, (96) p. Includes Addendum dated 27 May 1977, $43 \mathrm{p}$.

(2) Harris, F.R., 1976, "Geology of the MacMillan Tungsten Deposit," in Weiss, Alfred, editor, World Mining and Metals Technology, Proceedings of the Joint MMIJ-AIME Meeting, Denver, Colo.; AIME, New York, p. 65-79.

(3) Krohn, Douglas H., and Weist, Margaret Mlynarczyb, 1977, Principal Information on Montana Mines, Special Publication 75, Montana Bureau of Mines and Geology, $150 \mathrm{p}$.

(4) Larson, L.P., Lowrie, R.L., and Leland, G.R., 1971, Availability of Tungsten at Various Prices from Resources in the United States: U.S. Bur. Mines Inf. Circ. 8500,65 p.

(5) Laznicka, Peter, compiler, 1973, Manifile; the University of Manitoba File of Nonferrous Metal Deposits of the World: Hanitoba Univ., Dept. Earth Sci., Centre Precambrian Studies Pub. 2, 3 v.: Pt. 1 533 p.; Pt. 2, v. 1, 698 p.; Pt. 2, 767 p.

(6) Lemmon, D.M., 1969, "Tungsten," in Mineral and Water Resources of Utah - report of the United States Geological Survey in cooperation with Utah Geological and Mineralogical Survey and the Utah Water and Power Board prepared at the request of Senator Frank E. Moss of Utah of the Committee on Interior and Insular Affairs, United States Senate, Document No. 91-12, p.121-124.

(7) Lemmon, D.M., and Ross, D.C., 1956, "Resources," Chapter II in U.S. Business and Defense Services Administration Materials Survey Tungsten, compiled for the Office of Defense Mobilization by the U.S. Department of Commerce, Business and Defense Services Administration, p. II-1 - II-16.

(8) Ministerio das Minas e Energia (Brazil), 1969, Relatorio Preliminar Sobre as Investigacoes Geologicas na Mina Brejui-RN (Projeto Tungstenio/Molibdenio), Recife, Brazil, 53 p. (from Gus Goudarzi).

(9) Schruben, Paul, 1977, U.S. Geological Survey, Office of Resource Analysis, data collected from Northern Miner and State Department Reports. 
(10). U.S. Bureau of Mines, 1944, Iron Mounta in Tunsten Project, Sierra County, N. Mex., War Minerals Report No, 157, (January), 8 p.

(11) Wall Street Journal, 1977, Union Carbide advertisement, July 5, p. 7.

(12) Wright, Nancy, 1977, data from World Mineral Deposits Computer File, U.S. Geological Survey, Office of Resource Analysis. 
Mercury Deposits

Peterson, J.A., Bergquist, J.R., and Gassaway, J.S., 1977, Mercury in the Conterminous United States: Computerized Data File and Computer-Plotted Map of Occurrences, U.S. Geological Survey Open-File Report 77-859, 29 p.

Sainsbury, C.L. and MacKevett, E.M., Jr., 1965, Quicksilver Deposits of Southwestern Alaska, U.S. Geological Survey Bu17. 1187, 89 p.

U.S. Geological Survey, 1977, Computerized Resources Information Bank (CRIB), a mineral resources data file of the U.S. Geological Survey. 
(1) Naldrett, A.J., 1973, Nickel Sulfide Deposits - Their Classification and Genesis, with Special Emphasis on Deposits of Volcanic Association, Trans. Can. Inst. Min. Met., 76, 183-201.

(2) Annis, R.C., Cranstone, D.A., and Valleé, M. 1976, A Survey of Known Mineral Deposits in Canada That Are Not Being Mined, Canadian Dept. of Energy, Mines and Resources, Ottawa, (95) p.

(3) Douglas, R.J.W., ed. 1970, Geology and Economic Minerals of Canada, Economic Geology Report No. 1, Canadian Dept. of Energy, Mines and Resources, p. 838.

(4) Mackenzie, B.W., 1968, Nickel - Canada and the World, Min. Res. Div., Canadian Dept. of Energy, Mines and Resources, Ottawa, Min. Rept. 16, p. 176.

(5) Data from industry.

(6) Laznicka, Peter, compiler, 1973, Manifile; The University of Manitoba File of Nonferrous Metal Deposits of the World: Manitoba Univ., Dept. Earth Sci., Centre Precambrian Studies Pub. 2, 3 v.: Pt. 1, 533 p.; Pt. 2, v. 1, 698 p.; Pt. 2, v. 2, 767 p.

(7) U.S. Geological Survey, 1977, Computer Resources Information Bank (CRIB), a mineral resource data file of the U.S.G.S. 


\section{Vein Gold}

(1) Annis, R.C., Cranstone, D.A., and Vallée, M., 1975, A Survey of Known Mineral Deposits in Canada That Are Not Being Mined, Canadian Dept. of Energy, Mines and Resources, Ottawa, 96 p. Includes addendum dated 27 May 1977.

(2) Bostock, H.S., Mulligan, R. and Douglas, R.J.W., 1957, The Cordilleran Region, in Stockwell, C.H., ed., Geology and Economic Minerals of Canada, Geological Survey of Canada, p. 283-392.

(3) Laznicka, Peter, compiler, 1973, Manifile; The University of Manitoba File of NonFerrous Metal Deposits of the World: Manitoba Univ. Dept. Earth Sci., Centre Precambrian Studies Pub. 2, 3 v.: Pt. 1, 533 p.; Pt. 2, v. 1, 698 p.; Pt. 2, $767 \mathrm{p}$.

(4) Little, H.W., Belyea, H.R., Stott, D.F., Latour, B.A. and Douglas, R.J.W., 1968, Economic Minerals of Hestern Canada, in i Douglas, R.J.W., ed., Geology and Economic Minerals of Canada, Canadian Dept. of Energy, Mines and Resources, p. 489-546.

(5) Sinclair, W.D., Morin, J.A., Craig, D.B., and Marchand, M., 1976, Mineral Industry Report, 1975, Yukon Territory, Canadian Dept. of Indian and Northern Affairs, Ottawa, $210 \mathrm{p}$. 
Mafic Volcanogenic Copper

(1) Laznicka, Peter, compiler, 1973, Manifile; The University of Manitoba File of Nonferrous Metal Deposits of the Horld; Manitoba Univ., Dept. Earth Sci., Centre Precanbrian Studies Pub. 2, 3 v.: Pt. 1, 533 p.; Pt. 2, v. 1, 698p.; Pt. 2, $767 \mathrm{p}$. 


$$
\text { Felsic to Intermediate Volcanogenic }
$$

(1) Laznicka, Peter, compiler, 1973, manifile; the University of Manitoba File of Nonferrous Metal Deposits of the World: Manitoba Univ., Dept. Earth Sci., Centre Precambrian Studies Pub. 2, 3 v.: Pt. 1, 533 p.; Pt. 2, v. 1, 698 p.; Pt. 2, $767 \mathrm{p}$.

(2) U.S Geological Survey, 1977, Computer Resources Information Bank (CRIB), a mineral resources data file of the U.S.G.S. 


\section{Copper Skarn}

(1) Douglas, R.J.W., ed., 1970, Geology and Economic Minerals of Canada, Economic Geology Report No. 1, Dept. of Energy, Mines and Resources Canada, Geol. Survey of Canada, p. 838.

(2) Laznicka, Peter, compiler, 1973, Manifile; The University of Manitoba File of Nonferrous Metal Deposits of the World: Manitoba Univ., Dept. Earth Sci., Centre Precambrian Studies Pub. 2, 3 v.; Pt. 1, 533 p.; Pt. 2, v. 1, 698 p.; Pt. 2. v. 2, 767 p.

(3) Annis, R.C., Cranston, D.A., and Vallee, M., 1976, A Survey of Known Mineral Deposits in Canada That Are Not Being Mined, Canadian Dept. of Energy, Mines and Resources, Ottawa, 96 p. Includes addendum dated 27 May 1977.

(4) Pennzoil Corp., 1973, Prospectus dated 10 May 1973.

(5) Rosenkranz, R.D., Davidoff, R.L., and Lemons, J.F., Jr., 1979, Copper availability - Domestic, U.S. Bureau of Mines, IC 8809 , $31 \mathrm{p}$. 